Please do not remove this page

RMIT

UNIVERSITY

\title{
An overview of recent progress in the study of distributed multi-agent coordination
}

Cao, Yongcan; Yu, Wenwu; Ren, Wei; Chen, Guanrong

https://researchrepository.rmit.edu.au/esploro/outputs/9921859244401341/filesAndLinks?institution=61RMIT_INST\&index=null

Cao, Y., Yu, W., Ren, W., \& Chen, G. (2013). An overview of recent progress in the study of distributed multi-agent coordination. IEEE Transactions on Industrial Informatics, 9(1), 427-438.

https://doi.org/10.1109/TII.2012.2219061

Published Version: https://doi.org/10.1109/TII.2012.2219061

Repository homepage: https://researchrepository.rmit.edu.au

(C) 2005-2012 IEEE.

Downloaded On 2023/04/26 19:39:57 +1000

Please do not remove this page 
Thank you for downloading this document from the RMIT Research Repository.

The RMIT Research Repository is an open access database showcasing the research outputs of RMIT University researchers.

RMIT Research Repository: http://researchbank.rmit.edu.au/

\section{Citation:}

Cao, Y, Yu, W, Ren, W and Chen, G 2013, 'An overview of recent progress in the study of distributed multi-agent coordination', IEEE Transactions on Industrial Informatics, vol. 9, no. 1, pp. 427-438.

See this record in the RMIT Research Repository at:

http://researchbank.rmit.edu.au/view/rmit:20438

Version: Accepted Manuscript

Copyright Statement: (C) 2005-2012 IEEE.

Link to Published Version:

http://researchbank.rmit.edu.au/view/rmit:20438 


\title{
An Overview of Recent Progress in the Study of Distributed Multi-agent Coordination
}

\author{
Yongcan Cao, Member, IEEE, Wenwu Yu, Member, IEEE, \\ Wei Ren, Member, IEEE, and Guanrong Chen Fellow, IEEE
}

\begin{abstract}
This article reviews some main results and progress in distributed multi-agent coordination, with the focus on papers published in major control systems and robotics journals since 2006. Distributed coordination of multiple vehicles, including unmanned aerial vehicles (UAVs), unmanned ground vehicles (UGVs) and unmanned underwater vehicles (UUVs), has been a very active research subject studied extensively by the systems and control community. The recent results in this area are categorized into several directions, such as consensus, formation control, optimization, distributed task assignment, and estimation. After the review, a short discussion section is included to summarize the existing research and to propose several promising research directions along with some open problems that are deemed important therefore deserving further investigations.
\end{abstract}

\section{Index Terms}

Distributed coordination, formation control, sensor network, multi-agent system

\section{INTRODUCTION}

Control theory and practice may date back to the beginning of the last century when Wright Brothers attempted their first test flight in 1903. Since then, control theory has gradually gained popularity, receiving more and wider attention especially during the World War II when it was developed and applied to firecontrol systems, missile navigation and guidance, as well as various electronic automation devices. In

This work was supported by ......, and the Hong Kong RGC under GRF Grant CityU1114/11E.

Y. Cao and W. Ren are with the Department of Electrical and Computer Engineering, Utah State University, Logan, Utah 84322, USA. W. Yu is with the Department of Mathematics, Southeast University, Nanjing 210096, China. G. Chen is with the Department of Electronic Engineering, City University of Hong Kong, Hong Kong SAR, China.

Manuscript submitted to IEEE Transactions on Industrial Informatics on 31 July 2011. 
the past several decades, modern control theory was further advanced due to the booming of aerospace technology based on large-scale engineering systems.

During the rapid and sustained development of the modern control theory, technology for controlling a single vehicle, albeit higher-dimensional and complex, has become relatively mature and has produced many effective control tools such as PID control, adaptive control, nonlinear control, intelligent control, and robust control methodologies. In the past two decades in particular, control of multiple vehicles has received increasing demands spurred by the fact that many benefits can be obtained when a single complicated vehicle is equivalently replaced by multiple yet simpler vehicles. In this endeavor, two approaches are commonly adopted for controlling multiple vehicles: a centralized approach and a distributed approach. The centralized approach is based on a basic assumption that a central station is available and powerful enough to control a whole group of vehicles. Essentially, the centralized approach is a direct extension of the traditional single-vehicle-based control philosophy and strategy. On the contrary, the distributed approach does not require a central station for control, at the cost of becoming far more complex than the centralized one in structure and organization. Although both approaches are considered practical depending on the situations and conditions of the real applications, the distributed approach is believed more promising due to many inevitable physical constraints such as limited resources and energy, short wireless communication ranges, narrow bandwidths, and large sizes of vehicles to manage and control. Therefore, the focus of this overview is placed on the distributed approach.

In distributed control of a group of autonomous vehicles such as UAVs, UGVs and UUVs, the main objective typically is to have the whole group of vehicles working in a cooperative fashion throughout a distributed protocol. Here, cooperative refers to a close relationship among all vehicles in the group where information sharing plays a central role. The distributed approach has many advantages in achieving cooperative group performances, especially with low operational costs, less system requirements, high robustness, strong adaptivity, and flexible scalability, therefore has been widely recognized and appreciated.

The study of distributed control of multiple vehicles was perhaps first motivated by the work in distributed computing [1], management science [2], [3], and statistical physics [4]. In the control systems society, some pioneering works are generally referred to [5], [6], where an asynchronous agreement problem was studied for distributed decision-making problems. Thereafter, some consensus algorithms were studied under various information-flow constraints [7]-[11]. There are several journal special issues on the related topics published after 2006, including the IEEE Transactions on Control Systems Technology (vol. 15, no. 4, 2007), Proceedings of the IEEE (vol. 94, no. 4, 2007), ASME Journal of Dynamic 
Systems, Measurement, and Control (vol. 129, no. 5, 2007), SIAM Journal of Control and Optimization (vol. 48, no.1, 2009), and International Journal of Robust and Nonlinear Control (Vol. 21, no. 12, 2011). In addition, there are some more recent reviews and progress reports given in the surveys [12]-[15] and the books [16]-[21].

This article reviews some main results and recent progress in distributed multi-agent coordination, published in major control systems and robotics journals since 2006. For results before 2006, the readers are referred to [12]-[15].

Specifically, this article reviews the recent research results in the following directions, which are not independent but actually may have overlapping to some extent:

1. Consensus and the like (synchronization, rendezvous). Consensus refers to the group behavior that all the agents asymptotically reach a certain common agreement through a local distributed protocol, with or without predefined common speed and orientation.

2. Distributed formation and the like (flocking). Distributed formation refers to the group behavior that all the agents form a pre-designed geometrical configuration through local interactions with or without a common reference.

3. Distributed optimization. This refers to algorithmic developments for the analysis and optimization of large-scale distributed systems.

4. Distributed task assignment. This refers to the implementation of a task-assignment algorithm in a distributed fashion based on local information.

5. Distributed estimation and control. This refers to distributed control design based on local estimation about the needed global information.

The rest of this article is organized as follows. In Section II, basic notations of graph theory and stochastic matrices are introduced. Sections III, IV, V, VI, and VII describe the recent research results and progress in consensus, formation control, optimization, task assignment, and estimation, respectively. Finally, the article is concluded by a short section of discussions with future perspectives.

\section{PReliminaries}

This section introduces basic concepts and notations of graph theory and stochastic matrices.

\section{A. Graph Theory}

For a system of $n$ connected agents, its network topology may be modeled as a directed graph denoted $\mathcal{G}=(\mathcal{V}, \mathcal{W})$, where $\mathcal{V}=\left\{v_{1}, v_{2}, \cdots, v_{n}\right\}$ and $\mathcal{W} \subseteq \mathcal{V} \times \mathcal{V}$ are, respectively, the set of agents and the set 
of edges which directionally connect the agents together. Specifically, the directed edge denoted by an ordered pair $\left(v_{i}, v_{j}\right)$ means that agent $j$ can access the state information of agent $i$. Accordingly, agent $i$ is a neighbor of agent $j$. A directed path is a sequence of directed edges in the form of $\left(v_{1}, v_{2}\right),\left(v_{2}, v_{3}\right), \cdots$, with all $v_{i} \in \mathcal{V}$. A directed graph has a directed spanning tree if there exists at least one agent that has a directed path to every other agent. The union of a set of directed graphs with the same set of agents, $\left\{\mathcal{G}_{i_{1}}, \cdots, \mathcal{G}_{i_{m}}\right\}$, is a directed graph with the same set of agents and its set of edges is given by the union of the edge sets of all the directed graphs $\mathcal{G}_{i_{j}}, j=1, \cdots, m$. A complete directed graph is a directed graph in which each pair of distinct agents is bidirectionally connected by an edge, thus there is a directed path from any agent to any other agent in the network.

Two matrices are frequently used to represent the network topology: the adjacency matrix $\mathcal{A}=\left[a_{i j}\right] \in$ $\mathbb{R}^{n \times n}$ with $a_{i j}>0$ if $\left(v_{j}, v_{i}\right) \in \mathcal{W}$ and $a_{i j}=0$ otherwise, and the Laplacian matrix $\mathcal{L}=\left[\ell_{i j}\right] \in \mathbb{R}^{n \times n}$ with $\ell_{i i}=\sum_{j=1}^{n} a_{i j}$ and $\ell_{i j}=-a_{i j}, i \neq j$, which is generally asymmetric for directed graphs except complete directed graphs. The Laplacian $\mathcal{L}$ has at least one single zero eigenvalue with a corresponding eigenvector 1 consisting of all numeric 1 . Here and throughout, all matrices and vectors are assumed to have comparable dimensions unless otherwise indicated.

\section{B. Stochastic Matrices}

A nonnegative square matrix is called (row) stochastic matrix if its every row is summed up to one. The product of two stochastic matrices is still a stochastic matrix. A row stochastic matrix $P \in \mathbb{R}^{n \times n}$ is called indecomposable and aperiodic if $\lim _{k \rightarrow \infty} P^{k}=1 y^{T}$ for some $y \in \mathbb{R}^{n}$ [22].

\section{CONSEnsus}

Consider a group of $n$ agents, each with single-integrator kinematics described by

$$
\dot{x}_{i}(t)=u_{i}(t), \quad i=1, \cdots, n,
$$

where $x_{i}(t)$ and $u_{i}(t)$ are, respectively, the state and the control input of the $i$ th agent. A typical consensus control algorithm is designed as

$$
u_{i}(t)=\sum_{j=1}^{n} a_{i j}(t)\left[x_{j}(t)-x_{i}(t)\right],
$$

where $a_{i j}(t)$ is the $(i, j)$ th entry of the corresponding adjacency matrix at time $t$. The main idea behind (2) is that each agent moves towards the weighted average of the states of its neighbors. Given a switching network topology, coupling coefficients $a_{i j}(t)$ in (2), hence the graph topologies, are generally timevarying, due to the continuous motions of the dynamic agents. It is shown in [10], [11] that consensus is 
achieved if the underlying directed graph has a directed spanning tree in some jointly fashion in terms of a union of its time-varying graph topologies.

The idea behind consensus serves as a fundamental principle in the design of distributed multi-agent coordination algorithms. Therefore, investigating consensus has been a main research direction in the study of distributed multi-agent coordination. To bridge the gap between the study of consensus algorithms and many physical properties inherited in practical systems, it is necessary and meaningful to study consensus by considering many practical factors, such as actuation, control, communication, computation, and vehicle dynamics, which characterize some important features of practical systems. This is the main motivation to the study of consensus.

\section{A. Stochastic Network Topologies and Dynamics}

In multi-agent systems, the network topology among all vehicles plays a crucial role in determining consensus. The objective here is to explicitly identify necessary and/or sufficient conditions on the network topology such that consensus can be achieved under properly designed algorithms.

It is often reasonable to consider the case when the network topology is deterministic under ideal communication channels. Accordingly, main research on the consensus problem was conducted under a deterministic fixed/switching network topology. That is, the adjacency matrix $\mathcal{A}(t)$ is deterministic. Some other times, when considering random communication failures, random packet drops, communication channel instabilities inherited in physical communication channels, etc., it is necessary and important to study consensus problem in the stochastic setting where a network topology evolves according to some random distributions. That is, the adjacency matrix $\mathcal{A}(t)$ is stochastically evolving. This motivates the study of the consensus problem under a stochastic network topology.

In the deterministic setting, consensus is said to be achieved if all agents eventually reach agreement on a common state. In the stochastic setting, consensus is said to be achieved almost surely (respectively, in mean-square or in probability) if all agents reach agreement on a common state almost surely (respectively, in mean-square or with probability 1). Note that the problem studied in the stochastic setting is slightly different from that studied in the deterministic setting due to the different assumptions in terms of the network topology. Consensus over a stochastic network topology was perhaps first studied in [23], where some sufficient conditions on the network topology were given to guarantee consensus with probability 1 for systems with single-integrator kinematics (1), where the rate of convergence was also studied. Further results for consensus under a stochastic network topology were reported in [24][32], where research effort was conducted for systems with single-integrator kinematics [24]-[31] or 
double-integrator dynamics [32]. Consensus for single-integrator kinematics under stochastic network topology has been extensively studied, in particular, where some general conditions for almost-surely consensus was derived [26], [27], [30]. Loosely speaking, almost-surely consensus for single-integrator kinematics can be achieved, i.e., $x_{i}(t)-x_{j}(t) \rightarrow 0$ almost surely, if and only if the expectation of the network topology, namely, the network topology associated with the expectation $E[\mathcal{A}(t)]$, has a directed spanning tree. It is worth noting that the conditions are analogous to that in [10], [11], but in the stochastic setting. In view of the special structure of the closed-loop systems concerning consensus for single-integrator kinematics, the basic properties of the stochastic matrices play a crucial role in the convergence analysis of the associated control algorithms. Consensus for double-integrator dynamics was studied in [32], where the switching network topology is assumed to be driven by a Bernoulli process, and it was shown that consensus can be achieved if the union of all the graphs has a directed spanning tree. Apparently, the requirement on the network topology for double-integrator dynamics is a special case of that for single-integrator kinematics due to the difference nature of the final states (constant final states for single-integrator kinematics and possible dynamic final states for double-integrator dynamics) caused by the substantial dynamical difference. It is still an open question as if some general conditions (corresponding to some specific algorithms) exist for consensus with double-integrator dynamics.

Instead of focusing on analyzing the conditions on the network topology such that consensus can be achieved, a special type of consensus algorithm, the so-called gossip algorithm [33], [34], has been used to achieve consensus in the stochastic setting. Due to probabilistic pairwise communications, the gossip algorithm can always guarantee consensus almost surely if the available pairwise communication channels satisfy certain conditions (such as a connected graph or a graph with a directed spanning tree). The way of network topology switching does not play any role in the consideration of consensus.

The current study on consensus over stochastic network topologies has shown some interesting results regarding: (1) consensus algorithm design for various multi-agent systems, (2) conditions of the network topologies on consensus, and (3) effects of the stochastic network topologies on the convergence rate. Future research in this topic includes, but not limited to, the following two directions: (1) when the network topology itself is stochastic, how to determine the probability of reaching consensus almost surely? (2) compared with the deterministic network topology, what are the advantages and disadvantages of the stochastic network topology, regarding such as robustness and convergence rate?

As is well known, disturbances and uncertainties often exist in networked systems, for example, channel noise, communication noise, uncertainties in network parameters, etc. In addition to the stochastic network topologies discussed above, the effect of stochastic disturbances [35]-[40] and uncertainties [41], 
[42] on the consensus problem also needs investigation. Study has been mainly devoted to analyzing the performance of consensus algorithms subject to disturbances and to presenting conditions on the uncertainties such that consensus can be achieved. In addition, another interesting direction in dealing with disturbances and uncertainties is to design distributed local filtering algorithms so as to save energy and improve computational ability. Distributed local filtering algorithms play an important role and are more effective than traditional centralized filtering algorithms for multi-agent systems. For example, the authors of [43]-[45] designed some distributed Kalman filters to implement data fusion. In [46], by using the analysis of consensus and pinning control in synchronization of complex networks, the authors discussed distributed consensus filtering in sensor networks. Recently, Kalman filtering over a packetdropping network was designed through a probabilistic approach [47]. Today, it remains a challenging problem to incorporate both dynamics of consensus and probabilistic filtering (Kalman) into a unified methodology.

\section{B. Complex Dynamical Systems}

Since consensus is concerned with the behavior of a group of vehicles, it is natural to consider the system dynamics for practical vehicles in the study of the consensus problem. Although the study of consensus under various system dynamics is due to the existence of complex dynamics in practical systems, it is also interesting to observe that system dynamics play an important role in determining the final consensus state. For instance, the well-studied consensus of multi-agent systems with singleintegrator kinematics as in (1) often converges to a constant final value (i.e., a time function) instead. However, consensus for double-integrator dynamics might admit a dynamic final value. These important issues motivate the study of consensus under various system dynamics.

As a direct extension of the study of the consensus problem for systems with simple dynamics, for example, with single-integrator kinematics or double-integrator dynamics, consensus with general linear dynamics was also studied recently [48]-[53], where research is mainly devoted to finding feedback control laws such that consensus (in terms of the output states) can be achieved for general linear systems

$$
\dot{x}_{i}=A x_{i}+B u_{i}, \quad y_{i}=C x_{i},
$$

where $A, B$, and $C$ are constant matrices with compatible sizes. Apparently, the well-studied singleintegrator kinematics and double-integrator dynamics are special cases of (3) for properly choosing $A, B$, and $C$. 
As a further extension, consensus for complex systems has also been extensively studied. Here, the term consensus for complex systems is used for the study of consensus problem when the system dynamics are nonlinear [54]-[56], [56]-[87] or with nonlinear consensus algorithms [88]-[90]. Examples of the nonlinear system dynamics studied in the consensus problem include:

- Nonlinear oscillators [59]. The dynamics are often assumed to be governed by the Kuramoto equation

$$
\dot{\theta}_{i}=\omega_{i}+\frac{K}{N} \sum_{j=1}^{N} \sin \left(\theta_{j}-\theta_{i}\right), \quad i=1,2, \cdots, N,
$$

where $\theta_{i}$ and $\omega_{i}$ are, respectively, the phase and natural frequency of the $i$ th oscillator, $N$ is the number of oscillators, and $K$ is the control gain. Generally, the control gain $K$ plays a crucial role in determining the synchronizability of the network.

- Complex networks [57], [69]-[73], [82]-[84], [86], [91]. The dynamics are typically represented as

$$
\dot{x}_{i}(t)=f\left(x_{i}(t)\right)+c \sum_{j=1, j \neq i}^{N} a_{i j}(t) \Gamma\left(x_{j}(t)-x_{i}(t)\right), \quad i=1,2, \cdots, N,
$$

where $x_{i}=\left(x_{i 1}, x_{i 2}, \cdots, x_{i n}\right)^{T} \in \mathbb{R}^{n}$ is the state vector of the $i$ th node, $f: \mathbb{R}^{n} \mapsto \mathbb{R}^{n}$ is a nonlinear vector function, $c$ is the overall coupling strength, $A(t)=\left[a_{i j}(t)\right]$ is the outer coupling matrix with $a_{i j}(t)=1$ if node $i$ and node $j$ are connected at time $t$ but otherwise $a_{i j}(t)=0$, with $a_{i i}(t)=k_{i}$ (degree of node $i$ ), and $\Gamma$ is a general inner coupling matrix describing the inner interactions between different state components of agents. It is easy to see that model (1) with control input (2) is a special case of (5) with $f=0$.

- Nonholonomic mobile robots [56], [79], [85], [92]. The dynamics are described by

$$
\dot{x}_{i}=u_{i} \cos \theta_{i}, \quad \dot{y}_{i}=u_{i} \sin \theta_{i}, \quad \dot{\theta}_{i}=\omega_{i}, \quad i=1, \cdots, N
$$

where $\left[x_{i}, y_{i}\right]$ denotes the location of the $i$ th agent, and $u_{i}$ and $\omega_{i}$ denote, respectively, its translational and rotational velocity. Note that there are three states and two control inputs. Therefore, the dynamics for nonholonomic mobile robots are underactuated. This poses substantial difficulties in designing proper consensus algorithms with corresponding stability analysis.

- Rigid bodies and the like [65]-[68], [80], [81], [87]. One typical (but not unique) description of the dynamics is

$$
M_{i}\left(q_{i}\right) \ddot{q}_{i}+C_{i}\left(q_{i}, \dot{q}_{i}\right) \dot{q}_{i}+g_{i}\left(q_{i}\right)=\tau_{i}, \quad i=1, \cdots, N,
$$

where $q_{i} \in \mathbb{R}^{p}$ is the vector of generalized coordinates, $M_{i}\left(q_{i}\right) \in \mathbb{R}^{p \times p}$ is the symmetric positivedefinite inertia matrix, $C_{i}\left(q_{i}, \dot{q}_{i}\right) \dot{q}_{i} \in \mathbb{R}^{p}$ is the vector of Coriolis and centrifugal torques, $g_{i}\left(q_{i}\right)$ is 
the vector of gravitational torques, and $\tau_{i} \in \mathbb{R}^{p}$ is the vector of torques produced by the actuators associated with the $i$ th agent. In practice, the dynamics of many mechanical systems are similar to (7). A notable property regarding the dynamics of rigid bodies is that $\dot{M}_{i}\left(q_{i}\right)-2 C_{i}\left(q_{i}, \dot{q}_{i}\right)$ is skew-symmetric (i.e., $z^{T}\left[\dot{M}_{i}\left(q_{i}\right)-2 C_{i}\left(q_{i}, \dot{q}_{i}\right)\right] z=0$ for all $z \in \mathbb{R}^{p}$ ), which plays a crucial role in finding Lyapunov functions and the subsequent stability analysis.

Although the aforementioned system dynamics are different from the well-studied single-integrator kinematics and double-integrator dynamics, the main research problem is same, namely, to drive all agents to some common states through local interactions among agents. Similarly to the consensus algorithms proposed for systems with simple dynamics, the consensus algorithms used for these models are also based on a weighted average of the state differences, with some additional terms if necessary. Main research work has been conducted to design proper control algorithms and derive necessary and/or sufficient conditions such that consensus can be achieved ultimately.

Note that although the objective is same, i.e., to guarantee reaching agreement on some final states, the problem is more complicated due to the nonlinearity of the closed-loop systems. In addition, most properties of stochastic matrices cannot be directly applied to their convergence analysis. The main techniques used in their stability analysis include dissipativity theory [54], nonsmooth analysis [89], [92], [93], and especially Lyapunov functions [59], [65], [67], [68], [92], [94].

One particular interesting topic is synchronization in complex networks which has been widely investigated in the past decade [95], [96]. Mathematically, the definitions for synchronization in complex networks and consensus in multi-agent systems are very similar, so to differentiate these two definitions and promote research exchanges in these two topics, their differences are briefly summarized below.

1) Different Asymptotic States (Nonlinear Dynamics versus Linear Dynamics). In the studies of synchronization in complex networks, researchers focus on synchronization with self-nonlinear dynamics where each single system is unstable and thus the final asymptotic synchronization state is typically time-varying [95], [97]. However, in the investigations of multi-agent systems, the individual self-dynamics on each system is usually linear or zero and therefore the asymptotic consensus state is usually a constant [7], [9].

2) Different Focuses (Known Connectivity versus Time-varying Distributed Protocol). In synchronization of complex networks, the aim is to reveal how the network structure, which is known in priori, affects the nonlinear collective dynamics [95], [96], while the aim of consensus in multi-agent systems is to figure out how the designed distributed local protocol concerning mobile time-varying network structure affects the consensus behavior [7], [9], [10]. 
3) Different Approaches (Lyapunov Method versus Stochastic Matrix Theory). Since both complex networks and multi-agent systems are networked systems, algebraic graph theory [98] is a common approach to use. Because of the nonlinear terms in synchronization of complex networks, Lyapunov function method is usually used together with matrix theory [57], [91], [97]. In order to show consensus in multi-agent systems with time-varying network structures, stochastic matrix theory [5]-[7], [10] and convexity analysis [11] are often applied.

4) Different Inner Matrices $\Gamma$ (General Inner Matrix versus Particular Inner Matrix). In the typical simple consensus model, the inner matrices $\Gamma$ are usually an identity matrix and a rank-one matrix $\left(\begin{array}{ll}0 & 1 \\ 0 & 0\end{array}\right)$ for multi-agent systems with single-integrator kinematics [9] and double-integrator dynamics [99]-[101], respectively. In consensus models with higher-order dynamics [102], the inner matrix is similar. However, the inner matrix in system (5) is a general one.

In summary, synchronization in complex networks focuses on nonlinear dynamics while consensus in multi-agent systems focuses on distributed cooperative control, and thus different approaches are utilized.

The current research on consensus with complex systems focuses on fully-actuated systems although consensus for nonholonomic mobile robots [79], [85], [92], which is a typical underactuated system, has also been studied. Note that many mechanical systems are described by systems with underactuation. Therefore, it is important to develop appropriate consensus algorithms for underactuated systems.

\section{Delay Effects}

Time delay appears in almost all practical systems due to several reasons: (1) limited communication speed when information transmission exists; (2) measurement time required by the sensor to get the measurement information; (3) computation time required for generating the control inputs; and (4) execution time required for the inputs being acted. In general, time delay reflects an important property inherited in every practical systems due to actuation, control, communication, and computation.

Knowing that time delay might degrade the system performance or even destroy the system stability, studies have been conducted to investigate the effect of time delay on system performance and stability. A well-studied consensus algorithm for (1) is given in (2), where it is now assumed that time delay exists. Two types of time delay, communication delay and input delay, have been considered in the literature. Communication delay accounts for the time for information being transmitted from its origin to its destination. More precisely, if it takes time $T_{i j}$ for agent $i$ to receive information from agent $j$, the 
closed-loop system of (1) using (2) under a fixed network topology becomes

$$
\dot{x}_{i}(t)=\sum_{j=1}^{n} a_{i j}(t)\left[x_{j}\left(t-T_{i j}\right)-x_{i}(t)\right] .
$$

An interpretation of (8) is that at time $t$, agent $i$ receives information from agent $j$ and uses data $x_{j}\left(t-T_{i j}\right)$ instead of $x_{j}(t)$ due to the time delay. Note that agent $i$ can get its own information instantly, therefore, input delay can be considered as the summation of computation time and execution time. More precisely, if the input delay for agent $i$ is given by $T_{i}^{p}$, then the closed-loop system of (1) using (2) becomes

$$
\dot{x}_{i}(t)=\sum_{j=1}^{n} a_{i j}(t)\left[x_{j}\left(t-T_{i}^{p}\right)-x_{i}\left(t-T_{i}^{p}\right)\right] .
$$

Clearly, (8) refers to the case when only communication delay is considered while (9) refers to the case when only input delay is considered. It should be emphasized that both communication delay and input delay might be time-varying and they might co-exist at the same time.

In addition to time delay, it is also important to consider packet drops in exchanging state information. Fortunately, consensus with packet drops can be considered as a special case of consensus with time delay because re-sending packets after they were dropped can be easily done but just having time delay in the data transmission channels.

Thus, the main problem involved in consensus with time delay is to study the effects of time delay on the convergence and performance of consensus, refer to as consensusability [103].

Because time delay might affect the system stability, it is important to study under what conditions consensus can still be guaranteed even if time delay exists. In another word, can we find conditions on the time delay such that consensus can be achieved? For this purpose, several papers investigated the effect of time delay on the consensusability of (1) using (2). When there exists only (constant) input delay, a sufficient condition on the time delay to guarantee consensus under a fixed undirected interaction graph is presented in [9]. Specifically, an upper bound of the time delay is derived under which consensus can be achieved. This is a well-expected result because time delay normally degrades the system performance gradually and will not destroy the system stability unless the time delay is above certain threshold. Further studies can be found in, e.g., [104]-[114], which demonstrate that for (1) using (2), the communication delay does not affect the consensusability but the input delay does. In a similar manner, consensus with time delay was studied for systems with different dynamics, where the dynamics (1) are replaced by other more complex ones, such as double-integrator dynamics and the like [100], [115]-[122], complex networks [123]-[126], rigid bodies and the like [127], [128], and general nonlinear dynamics [129]. 
In summary, the existing study of consensus with time delay mainly focuses on analyzing the stability of consensus algorithms with time delays for various types of system dynamics, including linear and nonlinear dynamics. Generally speaking, consensus with time delay for systems with nonlinear dynamics is more challenging. For most consensus algorithms with time delays, the main research question is to determine an upper bound of the time delay under which time delay does not affect the consensusability. For communication delay, it is possible to achieve consensus under a relatively large time delay. A notable phenomenon in this case is that the final consensus state is constant. Considering the linear/nonlinear system dynamics in consensus, the main tools for stability analysis of the closed-loop systems include matrix theory [105], [106], Lyapunov functions [123], [124], frequency-domain approach [109], passivity [125], and the contraction principle [104].

Although consensus with time delay has been studied extensively, it is often assumed that time delay

is either constant or random. However, time delay itself might obey its own dynamics, which possibly depend on the communication distance, total computation load and computation capability, etc. Therefore, it is more suitable to represent the time delay as another system variable to be considered in the study of the consensus problem. In addition, it is also important to consider time delay and other physical constraints simultaneously in the study of the consensus problem.

\section{Sampled-data Framework}

The previous three subsections describe the main research work in the study of the consensus problem. The following introduces a few other aspects, namely, sampled-data framework, quantization, asynchronous effect, convergence speed, and finite-time convergence, that have been considered in the consensus problem as well. Among these topics, sampled-data framework, quantization, and asynchronous effects are considered due to some physical limitations in practical systems while convergence speed and finite-time convergence are concerned with the performance for some proposed consensus algorithms.

Due to the limitations in the measurement and control units, it is often impossible to acquire information measurements at an arbitrarily fast speed and to execute the control inputs instantaneously. Accordingly, the closed-loop systems are modeled in a hybrid fashion. That is, the system plant is described in a continuous-time setting while the measurements and control inputs are described in a piecewise constant fashion. For instance, in a sampled-data setting, (2) becomes

$$
u_{i}(t)=u_{i}(k T)=\sum_{j=1}^{n} a_{i j}(k T)\left[x_{j}(k T)-x_{i}(k T)\right], \quad k T \leq t<(k+1) T,
$$


where $T$ is the sampling period and $k$ is the discrete-time index. Essentially, (10) is a zero-order-hold version of $u_{i}(t)$ in the sense that the control inputs remain unchanged during each sampling period. Under this circumstance, consensus is studied in a sampled-data framework, called sampled-data consensus, which reflects the limitations inherited in physical measurement and control units. Meanwhile, it is also important to point out that the sampled-data consensus algorithms require much less information exchange and computational power than the continuous-time consensus algorithms. Accordingly, consensus under the sampled-data framework deserves certain consideration.

Sampled-data consensus was investigated in, e.g., [118], [130]-[134], [134]-[139]. Consensus for systems with single-integrator kinematics (1) was studied under a sampled-data framework with a fixed or a switching network topology, in [131], [132], where some necessary and/or sufficient conditions were presented to guarantee achieving consensus. Sampled-data consensus of systems with double-integrator kinematics was studied under fixed or switching network topologies in [118], [130], [133]-[138], [138], [139]. Due to the fact that an infinitely large sampling period will cause no information exchange among the agents, the main research question is to find conditions on the sampling period $T$, which might be time-varying, such that consensus can be achieved. The conditions on the network topology for the sampled-data closed-loop systems are mostly similar to that for the continuous-time closed-loop systems. Note that the existing research on consensus in a sampled-data framework mainly focuses on the simple system dynamics and thus the closed-loop system can be represented in terms of a linear matrix equation. The corresponding network stability can be analyzed by investigating the properties of the system matrices constructed based on the proposed consensus algorithms and the given network topology. Various approaches, including Lyapunov functions [130], [134], matrix theory [118], [135], [138], [139], stochastic matrices [136], and linear matrix inequalities [133], [134], have been adopted, and some necessary and/or sufficient conditions have been derived for guaranteeing sampled-data consensus.

It is natural to consider the sampled-data effect for consensus with general linear or nonlinear dynamics. In addition, it is meaningful to consider the case when all vehicles do not necessarily share the same sampling period or the sampling period is not necessarily constant. Accordingly, it is expected that a careful design of the sampling periods (associated with the proposed algorithms) might lead to the optimization of the closed-loop systems under the proposed algorithms subject to certain cost functions, such as maximum convergence rate and minimum total information exchange. In another word, it is intriguing to move from analysis to design when investigating the consensus problem in a sampled-data framework. 


\section{E. Asynchronous Effects}

In most existing research of the consensus problem, it is assumed that all agents update their states synchronously, which requires a synchronized clock for the whole group of agents. However, such a synchronized clock might not exist in real applications. This motivates the design of consensus algorithms in an asynchronous fashion; that is, each agent updates its own states regardless of the update times of other agents.

In most studies of the asynchronous consensus problem for networked systems, due to the intrinsic technical difficulties, usually only single-integrator kinematics (1) and double-integrator dynamics are considered. In [106], such an asynchronous consensus problem with time delay was investigated by utilizing some basic properties of stochastic matrices. Similarly in [140], the asynchronous consensus problem was studied by using matrix theory and graph theory, and in [141], by employing the paracontracting theory. In [142], the authors studied the asynchronous consensus problem for double-integrator dynamics and presented sufficient conditions to guarantee consensus, where a condition based on linear matrix inequalities was given.

Note that consensus in an asynchronous fashion has been considered mainly for single-integrator kinematics and double-integrator dynamics but not for other system dynamics. For certain linear systems, it might be expected that asynchronous communication does not affect the consensusability as shown in [106], [140] for single-integrator kinematics. However, a similar conclusion may not hold for systems with general dynamics, especially nonlinear dynamics. It is important to quantify the effects of the asynchronous communication on the consensus problem.

\section{F. Quantization}

Quantized consensus has been studied recently with motivation from digital signal processing. Here, quantized consensus refers to consensus when the measurements are digital rather than analog therefore the information received by each agent is not continuous and might have been truncated due to digital finite precision constraints. Roughly speaking, for an analog signal $s$, a typical quantizer with an accuracy parameter $\delta$, also referred as quantization step size, is described by

$$
Q(s)=q(s, \delta)
$$

where $Q(s)$ is the quantized signal and $q(\cdot, \cdot)$ is the associated quantization function. For instance, a quantizer rounding a signal $s$ to its nearest integer can be expressed as [143]

$$
Q(s)=n, \quad \text { if } s \in[(n-1 / 2) \delta,(n+1 / 2) \delta], \quad n \in \mathcal{Z},
$$


where $\mathcal{Z}$ denotes the integer set. Note that the type of quantizer might be different for different systems, hence $Q(s)$ may differ for different systems. Due to the truncation of the signals received, consensus is now considered achieved if the maximal state difference is not larger than the accuracy level associated with the whole system. A notable feature for consensus with quantization is that the time to reach consensus is usually finite. That is, it often takes a finite period of time for all agents' states to converge to an accuracy interval. Accordingly, the main research is to investigate the convergence time associated with the proposed consensus algorithm.

Quantized consensus was probably first studied in [143], where a quantized gossip algorithm was proposed and its convergence was analyzed. In particular, the bound of the convergence time for a complete graph was shown to be polynomial in the network size. In [144], coding/decoding strategies were introduced to the quantized consensus algorithms, where it was shown that the convergence rate depends on the accuracy of the quantization but not the coding/decoding schemes. In [145], [146], quantized consensus was studied via the gossip algorithm, with both lower and upper bounds of the convergence time derived in terms of the network size. Further results regarding quantized consensus were reported in [147]-[154], where the main research was also on studying the convergence time for various proposed quantized consensus algorithms as well as the quantization effects on the convergence time. It is intuitively reasonable that the convergence time depends on both the quantization level and the network topology. It is then natural to ask if and how the quantization methods affect the convergence time. This is an important measure of the robustness of a quantized consensus algorithm (with respect to the quantization method).

Note that it is interesting but also more challenging to study consensus for general linear/nonlinear systems with quantization. Because the difference between the truncated signal and the original signal is bounded, consensus with quantization can be considered as a special case of one without quantization when there exist bounded disturbances. Therefore, if consensus can be achieved for a group of vehicles in the absence of quantization, it might be intuitively correct to say that the differences among the states of all vehicles will be bounded if the quantization precision is small enough. However, it is still an open question to describe the quantization effects on consensus with general linear/nonlinear systems.

\section{G. Convergence Speed}

In addition to the study on the consensus problem with physical constraints mentioned in the previous subsections, it is also important to study the control performance of the consensus problem. From the control's perspective, it is natural to propose proper control algorithms and analyze the stability, and to 
optimize the proposed control algorithms under certain control performance indexes. In this subsection, the convergence speed problem is reviewed, which is an important performance measure for consensus algorithms.

For dynamics (1) using algorithm (2) in a connected undirected graph, the worst-case convergence speed was shown in [9] to be the Laplacian spectral gap:

$$
\min _{X \neq \mathbf{0}, \mathbf{1}^{T} X=0} \frac{X^{T} \mathcal{L} X}{\|X\|^{2}}=\lambda_{2}
$$

where $\mathbf{0}$ is an all-zero column vector, $X=\left[x_{1}, \cdots, x_{n}\right]^{T}, \mathcal{L}$ is the Laplacian matrix with $\lambda_{2}$ being smallest nonzero eigenvalue. Here, one should recall that the smallest eigenvalue of a Laplacian matrix for a connected undirected graph is zero and all the other eigenvalues are positive.

In order to increase the convergence speed, therefore, the above spectral gap should be enlarged. For this purpose, an iterative algorithm was proposed in [155] to maximize the above spectral gap, by employing a semidefinite programming solver.

Other than the smallest nonzero eigenvalue of the Laplacian matrix, another commonly used measure for the convergence speed is the following ratio, introduced in [156], [157]:

$$
\rho=\lim _{t \rightarrow \infty, X(t) \neq X^{\star}}\left(\frac{\left\|X(t)-X^{\star}\right\|}{\left\|X(0)-X^{\star}\right\|}\right)^{1 / t},
$$

where $X^{\star}$ represents the final equilibrium given by $\sigma \mathbf{1}$, where $\sigma$ is a constant.

In [156], this problem of finding the fastest convergence speed was casted into a semidefinite programming problem. Furthermore, the convergence speed defined by (14) was studied in both deterministic and stochastic settings. In the deterministic setting, it was studied in [157]-[159] with estimation of lower bounds. In the stochastic setting, this problem was studied in [23], [26], [160], with a per-step convergence factor introduced and discussed in [160], which itself can be considered a measure of the convergence speed.

The existing study mainly focuses on the analysis of the convergence speed under various network topologies and optimization of the convergence speed for certain given network topologies. Considering the fact that consensus under different network topologies may demonstrate different convergence speeds, a natural question arising to this topic is how to design an optimal (switching) network topology with proper adjacency matrix such that optimal convergence speed can be achieved.

\section{H. Finite-time Convergence}

As an extension of the study of convergence speed for the consensus problem, finite-time consensus, reaching consensus in a finite time, has also been studied recently. Compared with most existing research 
in the consensus problem, finite-time consensus demonstrates a disturbance rejection property and robustness against uncertainties. In addition, due to the finite-time convergence, it is often possible to decouple the consensus problem from other control objectives when they are considered simultaneously.

For a group of $n$ agents, e.g. with dynamics (1), the objective is to design $u_{i}(t)$ such that $x_{i}(t)=x_{j}(t)$ for $t \geq \bar{T}$, where $\bar{T}$ is a constant. Here, $\bar{T}$ is also called the consensus time.

Finite-time consensus for networked systems with single-integrator kinematics (1) in the continuoustime setting was solved in [88], [93], [161]-[164]. Finite-time consensus for networked systems with double-integrator dynamics in the continuous-time setting was studied in [165]. An important common characteristic of the proposed algorithms is the use of the signum function. It is well known that linear consensus algorithms can normally guarantee asymptotic convergence, but not finite-time convergence. On the contrary, the finite-time consensus algorithms developed in [88], [93], [161], [162], [165], which utilize the signum function, are able to do so.

Note that the existing research on finite-time consensus mainly focuses on systems with simple dynamics, such as single-integrator kinematics and double-integrator dynamics. Because many practical systems are better and more proper to be described by general linear/nonlinear dynamics, it is natural to study finite-time consensus for systems with general linear/nonlinear dynamics in the future.

\section{Remarks}

In summary, the existing research on the consensus problem has covered a number of physical properties for practical systems and control performance analysis. However, the study of the consensus problem covering multiple physical properties and/or control performance analysis has been largely ignored. In another word, two or more problems in the aforementioned subsections might need to be taken into consideration simultaneously when studying the consensus problem. In addition, consensus algorithms normally guarantee the agreement of a team of agents on some common states without taking any group formation into consideration. To reflect many practical applications where a group of agents are normally required to form some preferred geometric structure, it is desirable to consider a task-oriented formation control problem for a group of agents, which motivates the study of formation control presented in the next section.

\section{Formation CONTROL}

Compared with the consensus problem where the final states of all agents typically become a singleton, the final states of all agents can be more diversified under the formation control scenario. Indeed, 
formation control is more desirable in many practical applications such as formation flying, cooperative transportation, sensor networks, as well as combat intelligence, surveillance, and reconnaissance. In addition, the performance of a team of agents working cooperatively often exceeds the simple integration of the performances of all individual agents. For its broad applications and advantages, formation control has been a very active research subject in the control systems community, where a certain geometric pattern is aimed to form with or without a group reference. More precisely, the main objective of formation control is to coordinate a group of agents such that they can achieve some desired formation such that some tasks can be finished by the collaboration of the agents. Generally speaking, formation control can be categorized in terms of a group reference. Formation control without a group reference, called formation producing, refers to the algorithm design for a group of agents to reach some pre-desired geometric pattern in the absence of a group reference, which can also be considered as the control objective. Formation control with a group reference, called formation tracking, refers to the same task following the predesignated group reference. Due to the existence of the group reference, formation tracking is usually much more challenging than formation producing and control algorithms for the latter might not be useful for the former. As of today, there are still many open questions in solving the formation tracking problem.

In the following, recent research results and progress in formation control, including formation producing, formation tracking, and connectivity maintenance for consensus and formation control, are reviewed and discussed.

\section{A. Formation Producing}

The existing work in formation control aims at analyzing the formation behavior under certain control laws, along with stability analysis.

1) Matrix Theory: Considering the nature of multi-agent systems, matrix theory has been used frequently in the stability analysis of their distributed coordination.

Note that consensus input to each agent (see e.g., (2)) is essentially a weighted average of the differences between the states of the agent's neighbors and its own. As an extension of the consensus algorithms, some coupling matrices were introduced here to offset the corresponding control inputs by some angles [166][169]. For example, given the single-integrator kinematics in (1), the control input (2) is revised as

$$
u_{i}(t)=\sum_{j=1}^{n} a_{i j}(t) C\left[x_{j}(t)-x_{i}(t)\right]
$$


where $C$ is a coupling matrix with compatible size. If $x_{i} \in \mathbb{R}^{3}$, then $C$ can be viewed the 3-D rotational matrix. The main idea behind (15) is that the original control input for reaching consensus is rotated by some angles. The closed-loop system can be expressed in a vector form, whose stability can be determined by studying the distribution of the eigenvalues of a certain transfer matrix. Main research work was conducted in [166]-[169] to design proper algorithms and analyze the collective motions for systems with single-integrator kinematics and double-integrator dynamics.

Note that the collective motions for nonholonomic mobile robots were also studied recently, in, e.g., [170], [171]. Although the study in [166]-[169] is different from that in [170], [171], similarities exist in the sense that all agents will not move to the weighted average of the states of neighboring agents, but to some offsetted state. Noticeably, the offsetted state in [166]-[169] is properly designed, yet the offsetted state in [170], [171] is induced by the special nonlinear system dynamics.

In the study of formation producing with linear closed-loop systems, it is observed that the associated system matrix has two interesting properties: (1) the existence of at least one zero eigenvalue, and (2) the existence of at least one pair of eigenvalues on the imaginary axis. The two properties play an important role in the formation producing problem under a fixed network topology. However, the two properties might not be able to solve the formation producing problem under a switching network topology, which is still a challenging problem due to the complexity in the analysis of switching systems.

2) Lyapunov Function Approach: Although matrix theory is a relatively simple approach for stability analysis of the formation producing problem, it is not applicable in many formation producing scenarios, especially with nonlinear systems. It is then natural to consider the Lyapunov function approach, a powerful and efficient approach that has been used frequently to perform stability analysis.

By using the Lyapunov function approach, several typical formation producing scenarios have been studied, including the inverse agreement problem [172], leaderless flocking and stabilization [173]-[183], and circular formation alike [170], [171], [184]-[187]. In the inverse agreement problem [172], the objective is to force a team of agents to disperse in space. Roughly speaking, for the single-integrator kinematics (1), the corresponding control input has the form given by

$$
u_{i}(t)=\sum_{j=1}^{n} b_{i j}\left(\left\|x_{i}-x_{j}\right\|\right)\left[x_{i}(t)-x_{j}(t)\right],
$$

where $b_{i j}(\cdot)$ is a nonnegative function. Assuming that each agent can communicate with all other agents within a radius $R$, the agents will disperse in space with the relative distance between any pair of agents larger than $R$.

For the case of leaderless flocking, research has been conducted to stabilize a group of agents towards 
some desired geometric formation, where the inter-agent interaction is described directly or indirectly by some nonnegative potential function $V_{i j}\left(\left\|x_{i}-x_{j}\right\|\right)$ regardless of the final group velocity. Some notable properties for $V_{i j}\left(\left\|x_{i}-x_{j}\right\|\right)$ includes: (i) $V_{i j}\left(\left\|x_{i}-x_{j}\right\|\right)$ achieves its minimum when $\left\|x_{i}-x_{j}\right\|$ is equal to the desired inter-agent distance between agents $i$ and $j$, (ii) $V_{i j}\left(\left\|x_{i}-x_{j}\right\|\right)$ increases as $\left\|x_{i}-x_{j}\right\|$ decreases from the desired distance to zero and $V_{i j}\left(\left\|x_{i}-x_{j}\right\|\right)$ could approach infinity as $\left\|x_{i}-x_{j}\right\|$ approaches zero, and (iii) $V_{i j}\left(\left\|x_{i}-x_{j}\right\|\right)$ increases as $\left\|x_{i}-x_{j}\right\|$ increases from the desired distance to the maximum communication range. The basic idea behind the potential function $V_{i j}\left(\left\|x_{i}-x_{j}\right\|\right)$ is to drive the inter-agent distance to the desired value while avoiding possible inter-agent collision. The corresponding control law for each agent is usually chosen to be the same as the corresponding consensus algorithm except that the $x_{i}-x_{j}$ term is replaced by $\nabla_{x_{i}} V\left(\left\|x_{i}-x_{j}\right\|\right)$ here. A fundamental limitation is that all agents will normally converge to some (constant) inter-agent configuration locally in the sense that some nonnegative potential function achieves its local minimum. Accordingly, the inter-agent distance might not converge to the desired value globally. It is an interesting future research topic to ensure that the desired inter-agent distance can be achieved globally under properly designed control algorithms. In addition, the network topology associated with a team of agents is usually assumed to be undirected, which is not applicable to many practical systems which are directed.

For the case of circular formation and the like, the main research in [170], [171], [184], [186], [188] was devoted to the collective motion for nonhonolomic mobile robots with the dynamics given in (6). Denote $r_{i}=x_{i}+\iota y_{i}$, where $\iota=\sqrt{-1}$. Then (6) becomes

$$
\dot{r}_{i}=u_{i} e^{\iota \theta_{i}}, \quad \dot{\theta}_{i}=\omega_{i}, \quad i=1, \cdots, N .
$$

Due to the nature of the nonlinear dynamics, a consensus-like algorithm often renders a circular-like ultimate formation where the trajectories of all agents are circular and the relative phase difference (namely, $\theta_{i}-\theta_{j}$ ) is constant. The current work mainly focuses on the case when all agents share a common unit speed. Similar circular-like formation was analyzed in [185], [187], where the system dynamics are different from (6) but share a similar nonlinearity. Due to the nonlinearity of the system dynamics, it is a challenging task to incorporate time delay, disturbances, quantization, etc, into the existing research.

3) Graph Rigidity: For a network with a given number of agents, the edges are closely related to the shape of formation. Roughly speaking, if enough information regarding edge distances for a team of agents is available, the geometric structure of all agents is determined. Then the graph for the agents is rigid. According to [189], a graph of $n$ agents is rigid if at least $2 n-3$ edge distances are available. 
Motivated by the graph rigidity, research has been conducted in [190]-[194] to drive a group of agents to the desired inter-agent configuration by ensuring that a certain number of edge distances are identical to the desired ones. The graph rigidity recovery after loss of an agent has also been investigated. Compared with other formation producing algorithms which require edge vector information $\left(i . e ., x_{i}-x_{j}\right)$, less information is required in edge distance information (i.e., $\left.\left\|x_{i}-x_{j}\right\|\right)$. As a tradeoff, some unstable equilibria, such as a collinear initial configuration (the initial states of all agents are linearly dependent) and a common initial state (the initial states of all agents are identical), rather than the desired inter-agent configuration, might exist. In practical applications, it is important to design proper control algorithms such that a team of agents can avoid converging to the unstable equilibria.

4) Receding Horizon Approach: Receding horizon control (RHC), known also as model predictive control, has been introduced into the formation stabilization problem. By nature, RHC is a finite-horizon optimization problem. The employment of RHC in the formation stabilization problem is motivated by the fact that RHC is more capable of dealing with constraints.

The main research in this topic [195]-[197] has been devoted to deriving proper distributed control algorithms for a team of agents such that they can reach some desired formation by optimizing some finite-horizon cost functions in scenarios with or without time delay. Because RHC is essentially an optimization-based control strategy, the distributed control algorithms are typically given by solving optimization problems. Therefore, more computational time is required by RHC than other control approaches. Therefore, the potential computation-induced time delay needs to be taken into consideration in practical applications.

\section{B. Formation Tracking}

Although formation control without a group reference is interesting in theory, it is more realistic to study formation control in the presence of a group reference because it may represent a control objective or a common interest of the whole group. This scenario is now reviewed in this subsection.

1) Matrix Theory: Similarly to the case of formation producing, matrix theory is often used in the study of formation tracking problem.

An interesting problem in formation tracking is to design a distributed control algorithm to drive a team of agents to track some desired state. For example, given the single-integrator kinematics, control algorithms were designed in [198], [199], where the algorithms are similar to those consensus algorithms except that an extra term is introduced here due to the existence of the group reference. If properly designed, all agents can track the group reference accurately as reported in [198] while, with bounded 
tracking errors analyzed in [199], where a discretized version in [198] was considered. It is worth mentioning that the group reference can be arbitrarily chosen as long as its derivative is bounded. In [200][202], the synchronization of a group of linear systems to the output of another linear exosystem was investigated with or without parameter uncertainties. In [198], [199], a general group reference was discussed while in [200]-[202] a general system model was considered. How to solve formation tracking for general linear systems with a general group reference is still an open problem.

The formation tracking problem can be converted to a traditional stability problem by redefining the variables as the errors between each agent's state and the group reference. Then, the formation tracking problem is solved if the corresponding errors can be driven to zero. However, the formation producing problem, in general, cannot be solved in this way. Therefore, under a switching network topology, the formation tracking problem is generally easier than the formation producing problem.

2) Lyapunov Function Approach: Due to the broad applications of the Lyapunov function approach in the stability analysis, it has become an important tool in the study of the formation tracking problem as well.

Flocking with a dynamic group reference has been studied recently [203]-[206], where the objective is to design distributed control algorithms such that a team of agents move cohesively along the group reference. Compared with leaderless flocking, the study of flocking with a dynamic group reference is much more challenging both theoretically and technically. If enough information of the group reference is known, such as acceleration and/or velocity information of the group reference, flocking with a dynamic group reference can be solved by employing a gradient-based control law [203]-[205]. Another approach was proposed in [206], where a variable structure-based control law was used to solve the problem with less information required. Similarly to the study of the leaderless flocking problem, the existing research on flocking with a dynamic group reference can only reach a local minimization of certain potential functions because the potential function is generally unspecified but satisfies the conditions stated in Subsection IV-A. Accordingly, the inter-agent distance is not identical to the desired one. However, the potential based control can be easily designed to guarantee collision avoidance and maintain the initial inter-agent communication patterns. Nevertheless, it is still an open problem to consider the task with global inter-agent distance stabilization, collision avoidance, and initial communication pattern maintenance.

Formation control with a group reference was studied in both linear systems [207]-[209] and nonlinear systems [210]-[218] when the potential function $V\left(\left\|x_{i}-x_{j}\right\|\right)$ is replaced by some known functions, generally in the form of $\left\|x_{i}-x_{j}-d_{i j}\right\|^{2}$, where $d_{i j}$ denotes the desired distance between agents $i$ and $j$. 
Briefly, the nonlinear systems studied in this case include nonholonomic mobile robots (see (6)) [212][216], rigid bodies (see (7)) [217], [218], and linear systems with nonlinear terms [210], [211]. Compared with the flocking problem, the problem studied here is relatively easier due to the known $V\left(\left\|x_{i}-x_{j}\right\|\right)$. In general, the inter-agent distance can be driven to the desired one. As a tradeoff, the collision avoidance and initial communication pattern maintenance need to be considered separately.

\section{Connectivity Maintenance for Consensus and Formation Control}

In both consensus and formation control problems, it is often assumed that the network topology satisfies certain fundamental conditions, for example, is connected or has a directed spanning tree. However, a practical communication model is typically distance-based, i.e., two agents can communicate with each other if and only if their distance is smaller than a certain threshold, called communication range. This is particularly true for sensor networks. In order to guarantee consensus or formation control be achieved asymptotically, a connectivity maintenance mechanism is essential, which has been studied recently.

The main approach to maintaining the connectivity of a team of agents is to define some artificial potentials (between any pair of agents) in a proper way such that if two agents are neighbors initially then they will always communicate with each other thereafter [206], [219]-[228]. In general, the artificial potential between a pair of agents grows to be sufficiently large (could be unbounded) when the distance between them increases to be equal to the communication range. For properly designed control algorithms, which are usually composed of the gradients of the artificial potentials, the total artificial potential is nonincreasing. This then indicates that the initial communication patterns can be preserved because otherwise the total potential will become larger than the initial total artificial potential, as soon as some communication pattern is broken. Although this approach provides a systematic way to guarantee the connectivity, the corresponding control algorithms might require infinite large control inputs, which is not practical. Meanwhile, it is not even necessary to always maintain the initial communication patterns in order to guarantee the connectivity. Therefore, how to find a more effective way to guarantee connectivity deserves further investigation. In contrast to the studies in [206], [219]-[228], the authors in [229] investigated an interesting problem where the number of initially existing communication patterns plays a role in the connectivity maintenance for the consensus problem with single-integrator kinematics (1) and control input (2). Roughly speaking, if the initial graph is "sufficiently" connected in the sense that each agent has at least a certain number of neighbors, consensus can be guaranteed to be achieved. Note that the result can only be applied to systems with single-integrator kinematics therefore further investigation 
is expected for systems with high-order linear dynamics or nonlinear dynamics.

\section{Remarks}

Current research in formation control mainly focuses on a fixed formation where the inter-agent distance is fixed. Considering practical applications, however, it might require that the formation be adaptive with respect to the events performed by the team of agents. In addition, it is important to consider constraints, such as input saturation, quantization, and power limitation, in the formation control problem. Meanwhile, the robustness is another important factor that deserves considerable attention in real applications where noise and disturbances exist.

In terms of connectivity maintenance for consensus and formation control, research has been devoted to continuous-time systems but practical systems are more suitable to be modeled in a discrete-time setting, which makes the study of connectivity maintenance for discrete-time systems more meaningful. In general, the connectivity maintenance for discrete-time systems is more challenging due to the fundamental limitation of the corresponding control input, which is usually piecewise constant rather than continuous.

\section{OPTIMIZATION}

Optimization is an important subject in the studies of control systems. The main objective of optimization is to find an optimal strategy subject to some given constraints such as cost functions. Recently optimization in distributed multi-agent coordination has been studied concerning convergence speed and some specific cost functions, which are respectively reviewed below.

\section{A. Convergence Speed}

As discussed above, one important problem in consensus is the convergence speed, which characterizes how fast consensus can be achieved therefore is desirable to optimize. In this regard, the convergence speed is the cost function to be optimized.

Consider a group of $n$ agents with dynamics described by the single-integrator kinematics (1). Equipped with (2), the dynamical equation (1) can be written in a matrix form, as

$$
\dot{X}(t)=-\mathcal{L} X(t)
$$

where $X(t)=\left[x_{1}(t), \cdots, x_{n}(t)\right]^{T}$ and $\mathcal{L}$ is the Laplacian matrix. For a network with a fixed topology, $\mathcal{L}$ is a constant matrix. 
Motivated by the observation that the smallest nonzero eigenvalue of the Laplacian matrix, $\lambda_{2}(\mathcal{L})$, determines the worst-case convergence speed [9], research has been conducted to maximize the convergence speed [155], [230] by choosing optimal weights associated with edges. In contrast to [155], [230], where the systems are assumed to have single-integrator kinematics, optimization of the convergence speed for double-integrator dynamics was considered in [231], where the convergence speed is defined in a similar way to the $\lambda_{2}(\mathcal{L})$ for the case with single-integrator kinematics. It is worth mentioning that optimal convergence for general linear systems and nonlinear systems is still an open problem.

As mentioned above, other than $\lambda_{2}(\mathcal{L})$, another commonly used measure for the convergence speed is given by (14). The corresponding optimization problem is

$$
\max _{u_{i}(t)} \rho,
$$

where $\rho$ is defined in (14). Existing research in [156], [232] focuses on the case when all agents converge to the average of the initial states, i.e., $X^{\star}=\left[\frac{1}{n} \sum_{i=1}^{n} x_{i}(0)\right] 1$. For an arbitrary fixed or switching network topology, the optimization problem (19) is challenging if $X^{\star}$ is unknown. But if $X^{\star}$ is chosen as $\left[\frac{1}{n} \sum_{i=1}^{n} x_{i}(t)\right] \mathbf{1}$, then the problem becomes much easier.

\section{B. Specific Cost Functions}

In addition to the fastest convergence speed requirement, various cost functions are also subject to minimization.

One interesting problem studied in this setting is distributed multi-agent optimization, which is motivated by solving one challenge in wireless sensor networks, namely, to estimate the environment parameters and/or some functions of interest, such as temperature and source location [233]. As a simple strategy, each sensor node can send its data to some existing central location which can then process the data if the central location is sufficiently powered. However, due to the limited power resources and communication capabilities, this strategy is often not applicable. An alternative approach to achieving a similar objective is to estimate the environment parameters and/or some functions of interest locally, which requires much less communication bandwidth and power. In wireless sensor networks [233], the estimation problem is usually modeled as a distributed multi-agent optimization problem. Roughly speaking, the objective of distributed multi-agent optimization is to cooperatively minimize the cost function

$$
\sum_{i=1}^{n} f_{i}(x)
$$

where the function $f_{i}: \mathbb{R}^{n} \mapsto \mathbb{R}$ represents the cost of agent $i$, known by this agent only, and $x \in \mathbb{R}^{m}$ is a decision vector. In [233], an incremental subgradient approach was used to solve the optimization 
problem for a ring type of network. It should be noted that [233] does not provide much discussion on the optimization problem under other types of network topologies.

Ref. [234] was probably the first paper studying the distributed multi-agent optimization problem under a consensus-based framework. The problem considered therein is formulated as

$$
\begin{array}{ll}
\operatorname{minimize} & \sum_{i=1}^{n} f_{i}(x) \\
\text { s.t. } & x \in \mathbb{R}^{n},
\end{array}
$$

where each $f_{i}: \mathbb{R}^{n} \rightarrow \mathbb{R}$ is assumed to be a convex function. Inspired by the average consensus algorithm and the standard subgradient method, a consensus-like algorithm was proposed as

$$
x_{i}(k+1)=\sum_{j=1}^{n} a_{i j}(k) x_{j}(k)-\alpha g_{i}\left(x_{i}(k)\right)
$$

where $\alpha$ is the step size and $g_{i}\left(x_{i}(k)\right)$ is the subgradient of $f_{i}(x)$ at $x=x_{i}(k)$. In [233], $\sum_{j=1}^{n} a_{i j}(k) x_{j}(k)$ in (20) was replaced by $x_{i-1}(k)$ with $x_{0}(k)=x_{n}(k-1)$. Note that the algorithm (20) can only find sub-optimal solutions, determined by the constant step size $\alpha$. Further results in this topic can be found in [235]-[237], where a similar distributed multi-agent optimization problem was studied within various scenarios, such as under constraints [235], over random networks [236], and with broadcast-based communications in an asynchronous setting [237]. In the existing research, time delay and disturbances have not been taken into consideration. Therefore, it is important to consider time delay and disturbances in the distributed multi-agent optimization problem due to their wide existence in wireless sensor networks.

In addition to the distributed multi-agent optimization problem where the cost function is a sum of a series of convex functions, distributed optimization has also been considered for both infinite-horizon cost functions [238]-[242] given by

$$
J_{i}=\int_{0}^{\infty}\left[X^{T}(t) Q X(t)+U^{T}(t) R U(t)\right] \mathrm{d} t
$$

and finite-horizon cost functions [243]-[246] given by

$$
J_{f}=\int_{0}^{t_{f}}\left[X^{T}(t) Q X(t)+U^{T}(t) R U(t)\right] \mathrm{d} t,
$$

where $X \in \mathbb{R}^{n}$ is the state, $U \in \mathbb{R}^{n}$ is the control input, and $t_{f}$ is a positive constant. It is worth mentioning that the RHC approach discussed in Section IV-A typically has finite-horizon cost functions. Different from the research reported in [234]-[237], which is to find the optimal estimated state, the objective here is to find the optimal control laws subject to the minimization of certain cost functions. Due to requirements of optimizing the cost functions when designing the control laws, the computational complexity becomes an important problem to study. Meanwhile, the network topology plays a significant 
role in the optimization problem with certain cost functions, therefore it is also useful to optimize the network topology subject to certain cost functions.

\section{Distributed TAsk Assignment}

Distributed task assignment refers to the study of task assignment of a group of dynamical agents in a distributed manner, which can be roughly categorized into coverage control, scheduling, and surveillance. Compared with the previous studies discussed in Sections III, IV, and V, distributed task assignment focuses on the three task-oriented research problems, where each topic has its unique features.

\section{A. Coverage Control}

Coverage control is an active research direction in mobile sensor networks, where the objective is to properly assign the mobile sensors' motion in order to maximize the detection probability.

Let $Q$ be a convex space with $\phi$ represent the distribution density function which indicates the probability that some event takes place over $Q$ [247]. Consider a group of $n$ mobile sensors whose locations are specified by $P=\left[p_{1}, \cdots, p_{n}\right]$. The sensor performance at a point $q$ degrades with respect to the distances $\left\|q-p_{i}\right\|$, which are all described by a nondecreasing differentiable function, $f$. The coverage control problem is to find a local controller for each mobile sensor such that the following cost function is minimized:

$$
J=\sum_{i=1}^{n} \int f\left(\left\|q-p_{i}\right\|\right) \phi(q) d q .
$$

This coverage control by nature is an optimization problem. Main research in this topic was reported in, e.g., [248]-[255], where the coverage control problem was considered in two directions, namely, analysis of coverage control under various practical constraints, such as limited sensing/communication capacities [248], load balancing [254], and nonholonomic mobile robots [255], and algorithms for coverage control [251]. Noting that time delay and uncertainties have not been considered in the coverage control problem, it is interesting to consider the effect of time delay and uncertainties in the coverage control problem. Moreover, the density function $\phi$ might be time-dependent in real systems, which is another interesting research topic for further study.

\section{B. Scheduling}

Another interesting topic in distributed task assignment is distributed scheduling, which refers to the scheduling of a group of dynamical agents in a distributed manner. Distributed scheduling has many 
potential applications in military and civilian sectors, and can be roughly categorized into two typical problems, namely sequence optimization [256] and task allocation [257]-[261]. The objective of sequence optimization is to schedule a team of agents such that some metrics can be optimized. For instance, in [256], an optimal scheduling sequence was designed to fuel a group of UAVs via dynamic programming, where the metric is the total spending time. The objective of task allocation is to distribute certain number of tasks to a team of agents such that they can balance the total tasks. If the number of tasks for each agent is considered a variable in the consensus problem, the task allocation might be viewed as a consensus problem except that a limitation on the total number of tasks for all agents exists. A notable feature of the distributed task assignment problem is that various constraints may exist due to the physical properties associated with the agents. In view of the difference objectives for sequence optimization and task allocation, it is an interesting topic to consider combining both objectives of the two problems simultaneously.

\section{Surveillance}

Distributed surveillance means to monitor a certain area by using a group of mobile agents in distributed coordination. Distributed surveillance has a number of potential applications, such as board security guarding, forest fire monitoring, and oil spill patrolling.

The main motivation of distributed surveillance is that a team of agents can monitor a given (large) area more effectively than a single agent when the team of agents works in a cooperative fashion. Accordingly, an important research problem in distributed surveillance is to design environment-based cooperative control laws for all coordinated agents such that the given area can be monitored efficiently. Recent research in distributed surveillance has been reported in [262]-[269], where a number of physical limitations were identified and considered such as time delay and uncertainties [265], [268], collision avoidance between agents [266], and heterogeneously distributed agents [268]. The current research is conducted under the assumption that each agent has enough power such that any designed control law can be applied. However, due to the power constraints, each agent might be subjected to constraints such as bounded control input, limited distance to travel, and finite accuracy level, etc., therefore it is interesting and important to consider these limitations in the distributed surveillance problem in the future.

\section{ESTIMATION}

Due to the absence of global information, used for achieving group coordination in many cases, a distributed estimation scheme is needed for estimation. The first problem is to design local distributed 
estimators such that some global information can be estimated asymptotically or in finite time. The second problem is to design local controllers based on the local estimators such that the closed-loop system is stable. The estimation-based distributed control is essentially a combination of both centralized control and distributed control in such a way that distributed control is used in the estimation of some global information and the centralized control is used in the local controllers design. The estimation-based distributed control strategy often inherits the merits of both centralized control and distributed control. However, it is worth emphasizing that a closed-loop system with distributed estimators is much more complicated to design than one without distributed estimators.

Main research in this topic has been reported in [163], [270]-[275], where the joint estimation and control problem was considered subject to disturbances [270], [272], [273] or without disturbances [163], [271], [274], [275]. In [163], [270]-[275], a joint estimation and control problem is solved in the sense that the distributed estimator is used in the design of proper control algorithms such that certain global objective can be achieved. Without the aid of distributed estimators, the control design is very hard and even impossible. As can be noticed from [276]-[282], the distributed estimation problem has been considered without much discussion on specific control problems. In general, the joint estimation and control idea has been an important approach in the study of distributed multi-agent coordination where only neighbor-based information is not sufficient for the controllers design. On the other hand, in real applications properly designed distributed estimators might be used to replace some expensive sensor.

In general, it remains a challenging problem to study task-oriented coordination control systems where the use of distributed estimation is either necessary or an appropriate replacement of certain expensive measurement devices, at the costs of difficult control system design and complex system stability analysis. Moreover, physical limitations such as bounded control input, asynchronous communication, and information quantization, could potentially enhance the applicability of the joint estimation and control scheme in various distributed multi-agent coordination systems.

\section{DISCUSSION}

This article has reviewed some recent research and development in distributed multi-agent coordination. In addition to the theoretical results reviewed above, many experiments were also conducted to validate the theoretical designs and analysis, as can be found in [283]-[288], to name just a few representative reports. Although the existing theoretical research and experiments have solved a number of technical problems in distributed multi-agent coordination, there are still many interesting, important and yet challenging research problems deserving further investigation. Some of them are briefly summarized as follows: 
- Quantization effects in distributed coordination algorithms. The current research efforts focus on studying distributed coordination problems with control inputs and measurements being analog signals with continuous values. However, digital signal processing techniques require digital inputs and sampled-data measurements. Although quantization effects have been studied in several coordination problems, the quantization effect in some other distributed coordination problems remain unsolved and even untouched.

- Optimization with integrated individual and global cost functions. Optimization problem in distributed coordination has been studied with various cost functions. In real systems, each individual agent has both local and global objectives, contributing to an integration of both individual and global cost functions. Therefore, optimizing a combined objective is more realistic but also more challenging. Another interesting problem is to investigate how to balance the individual cost functions and the global cost function toward a common objective.

- Intelligent coordination. Intelligent coordination refers to the distributed coordination of a team of agents in the presence of (artificial) intelligence, namely, each agent is intelligent, therefore can choose the best possible responses based on its own objective. Intelligent coordination has potential applications not only in engineering and technology but also in economics and social studies. Although research problems, such as pursuer-invader problem [289]-[292] and the game theory in distributed coordination [293]-[298], have been studied recently, there are still many open questions, especially the understanding of group behaviors in the presence of agent intelligence. One interesting problem is how to interpret the underlying complex networks as well as to stabilize and optimize the network in the presence of agent intelligence.

- Competition and cooperation. Today, most research is conducted based on local cooperation but not competition. This posts an obvious limitation because competition not only exists but also plays an positive role in group coordination. For example, due to the lack of competition, the final states of the traditional consensus algorithms are always limited to be within some region in the state space determined by the initial agent states. One interesting question is how to introduce competition into distributed coordination so as to arrive at different regions and to improve the system performance that rewards different agents with different benefits.

- Centralization and decentralization. Although decentralization shows obvious advantages over centralization, such as scalability and robustness, decentralization also has its own drawbacks. One shortcoming is that, under decentralized protocols, some agents cannot predict the group behavior based only on the available local information. Consequently, some group behavior cannot be con- 
trolled. As a sensible example, current economic crisis actually illustrates some disadvantages of behavioral decentralization. One interesting question, therefore, is how to balance decentralization and centralization so as to further improve the overall systems performance.

\section{REFERENCES}

[1] N. A. Lynch, Distributed Algorithms. San Francisco, California: Morgan Kaufmann Publishers, Inc., 1996.

[2] M. H. DeGroot, "Reaching a consensus," Journal of American Statistical Association, vol. 69, no. 345, pp. 118-121, 1974.

[3] R. L. Winkler, "The consensus of subjective probability distributions," Manage Science, vol. 15, no. 2, pp. B61-B75, October 1968.

[4] T. Vicsek, A. Czirok, E. B. Jacob, I. Cohen, and O. Schochet, "Novel type of phase transitions in a system of self-driven particles," Physical Review Letters, vol. 75, no. 6, pp. 1226-1229, 1995.

[5] J. N. Tsitsiklis, "Problems in decentralized decision making and computation," Ph.D. dissertation, MIT, 1984.

[6] J. N. Tsitsiklis and M. Athans, "Guaranteed robustness properties of multivariable nonlinear stochastic optimal regulators," IEEE Transactions on Automatic Control, vol. 29, no. 8, pp. 690-696, August 1984.

[7] A. Jadbabaie, J. Lin, and A. S. Morse, "Coordination of groups of mobile autonomous agents using nearest neighbor rules," IEEE Transactions on Automatic Control, vol. 48, no. 6, pp. 988-1001, June 2003.

[8] J. A. Fax and R. M. Murray, "Information flow and cooperative control of vehicle formations," IEEE Transactions on Automatic Control, vol. 49, no. 9, pp. 1465-1476, September 2004.

[9] R. Olfati-Saber and R. M. Murray, "Consensus problems in networks of agents with switching topology and time-delays," IEEE Transactions on Automatic Control, vol. 49, no. 9, pp. 1520-1533, September 2004.

[10] W. Ren and R. W. Beard, "Consensus seeking in multiagent systems under dynamically changing interaction topologies," IEEE Transactions on Automatic Control, vol. 50, no. 5, pp. 655-661, May 2005.

[11] L. Moreau, "Stability of multi-agent systems with time-dependent communication links," IEEE Transactions on Automatic Control, vol. 50, no. 2, pp. 169-182, February 2005.

[12] N. E. Leonard, D. A. Paley, F. Lekien, R. Sepulchre, D. M. Fratantoni, and R. E. Davis, "Collective motion, sensor networks, and ocean sampling," Proceedings of the IEEE, vol. 95, no. 1, pp. 48-74, January 2007.

[13] W. Ren, R. W. Beard, and E. M. Atkins, "Information consensus in multivehicle cooperative control: Collective group behavior through local interaction,” IEEE Control Systems Magazine, vol. 27, no. 2, pp. 71-82, April 2007.

[14] R. M. Murray, "Recent research in cooperative control of multivehicle systems," ASME Journal of Dynamic Systems, Measurement, and Control, vol. 129, no. 5, pp. 571-583, September 2007.

[15] R. Olfati-Saber, J. A. Fax, and R. M. Murray, "Consensus and cooperation in networked multi-agent systems," Proceedings of the IEEE, vol. 95, no. 1, pp. 215-233, January 2007.

[16] W. Ren and R. W. Beard, Distributed Consensus in Multi-vehicle Cooperative Control, ser. Communications and Control Engineering. London: Springer-Verlag, 2008.

[17] F. Bullo, J. Cortés, and S. Martínez, Distributed Control of Robotic Networks, ser. Applied Mathematics Series. Princeton University Press, 2009.

[18] Z. Qu, Cooperative Control of Dynamical Systems: Applications to Autonomous Vehicles. Springer-Verlag, 2009.

[19] M. Mesbahi and M. Egerstedt, Graph Theoretic Methods for Multiagent Networks. Princeton University Press, 2010. 
[20] W. Ren and Y. Cao, Distributed Coordination of Multi-agent Networks: Emergent Problems, Models, and Issues, ser. Communications and Control Engineering. London: Springer-Verlag, 2011.

[21] H. Bai, M. Arcak, and J. Wen, Cooperative Control Design: A Systematic, Passivity-Based Approach, ser. Communications and Control Engineering. New York: Springer-Verlag, 2011.

[22] J. Wolfowitz, "Products of indecomposable, aperiodic, stochastic matrices," Proceedings of the American Mathematical Society, vol. 15, pp. 733-736, 1963.

[23] Y. Hatano and M. Mesbahi, “Agreement over random networks," IEEE Transactions on Automatic Control, vol. 50, no. 11, pp. 1867-1872, November 2005.

[24] C. W. Wu, "Synchronization and convergence of linear dynamics in random directed networks," IEEE Transactions on Automatic Control, vol. 51, no. 7, pp. 1207-1210, July 2006.

[25] M. Porfiri and D. J. Stilwell, “Consensus seeking over random weighted directed graphs,” IEEE Transactions on Automatic Control, vol. 52, no. 9, pp. 1767-1773, September 2007.

[26] M. Akar and R. Shorten, "Distributed probabilistic synchronization algorithms for communication networks," IEEE Transactions on Automatic Control, vol. 53, no. 1, pp. 389-393, February 2008.

[27] A. Tahbaz-Salehi and A. Jadbabaie, "A necessary and sufficient condition for consensus over random networks," IEEE Transactions on Automatic Control, vol. 53, no. 3, pp. 791-795, April 2008.

[28] M. Huang, S. Dey, G. N. Nair, and J. H. Manton, "Stochastic consensus over noisy networks with markovian and arbitrary switches," Automatica, vol. 46, no. 10, pp. 1571-1583, 2010.

[29] M. Huang and J. H. Manton, "Stochastic consensus seeking with noisy and directed inter-agent communication: Fixed and randomly varying topologies," IEEE Transactions on Automatic Control, vol. 55, no. 1, pp. 235-241, January 2010.

[30] A. Tahbaz-Salehi and A. Jadbabaie, "Consensus over ergodic stationary graph processes," IEEE Transactions on Automatic Control, vol. 55, no. 1, pp. 225-230, January 2010.

[31] G. Yin, Y. Sun, and L. Y. Wang, "Asymptotic properties of consensus-type algorithms for networked systems with regime-switching topologies," Automatica, vol. 47, no. 7, pp. 1366-1378, 2011.

[32] Y. Zhang and Y.-P. Tian, "Consentability and protocol design of multi-agent systems with stochastic switching topology," Automatica, vol. 45, no. 5, pp. 1195-1201, 2009.

[33] S. Boyd, A. Ghosh, B. Prabhakar, and D. Shah, "Randomized gossip algorithms," IEEE Transactions on Information Theory, vol. 52, no. 6, pp. 2508-2530, 2006.

[34] A. G. Dimakis, S. Kar, J. M. F. Moura, M. G. Rabbat, and A. Scaglione, "Gossip algorithms for distributed signal processing," Proceedings of the IEEE, vol. 98, no. 11, pp. 1847-1864, November 2010.

[35] T. Li and J.-F. Zhang, "Mean square average-consensus under measurement noises and fixed topologies: necessary and sufficient conditions," Automatica, vol. 45, no. 8, pp. 1929-1936, 2009.

[36] Y. Liu and Y. Jia, " $H_{\infty}$ consensus control of multi-agent systems with switching topology: a dynamic output feedback protocol," International Journal of Control, vol. 83, no. 3, pp. 527-537, 2010.

[37] — "Consensus problem of high-order multi-agent systems with external disturbances: An $H_{\infty}$ analysis approach," International Journal of Robust and Nonlinear Control, vol. 20, no. 14, pp. 1579-1593, 2010.

[38] Z. Li, Z. Duan, and G. Chen, "On $H_{\infty}$ and $H_{2}$ performance regions of multi-agent systems," Automatica, vol. 47, no. 4, pp. 797-803, 2011.

[39] A. Garulli and A. Giannitrapani, "Analysis of consensus protocols with bounded measurement errors," Systems and Control Letters, vol. 60, no. 1, pp. 44-52, 2011. 
[40] H. Yang, Z. Zhang, and S. Zhang, "Consensus of second-order multi-agent systems with exogenous disturbances," International Journal of Robust and Nonlinear Control, vol. 21, no. 9, pp. 945-956, 2011.

[41] Q. Hui, W. M. Haddad, and S. P. Bhat, "On robust control algorithms for nonlinear network consensus protocols," International Journal of Robust and Nonlinear Control, vol. 20, no. 3, pp. 269-284, 2010.

[42] H. Kim, H. Shim, and J. H. Seo, "Output consensus of heterogeneous uncertain linear multi-agent systems," IEEE Transactions on Automatic Control, vol. 56, no. 1, pp. 200-206, January 2011.

[43] R. Olfati-Saber, "Distributed kalman filter with embedded consensus filters," in Proceedings of the IEEE Conference on Decision and Control, Seville, Spain, December 2005, pp. 8179-8184.

[44] R. Olfati-Saber and J. S. Shamma, “Consensus filters for sensor networks and distributed sensor fusion," in Proceedings of the IEEE Conference on Decision and Control, Seville, Spain, December 2005, pp. 6698-6703.

[45] R. Olfati-Saber, "Distributed kalman filtering for sensor networks," in Proceedings of the IEEE Conference on Decision and Control, New Orleans, LA, USA, December 2007, pp. 5492-5498.

[46] W. Yu, G. Chen, Z. Wang, and W. Yang, "Distributed consensus filtering in sensor networks," IEEE Transactions on Systems, Man, and Cybernetics, Part B: Cybernetics, vol. 39, no. 6, pp. 1568-1577, 2009.

[47] L. Shi, M. Epstein, and R. M. Murray, "Kalman filtering over a packet-dropping network: a probabilistic perspective," IEEE Transactions on Automatic Control, vol. 55, no. 3, pp. 594-604, 2010.

[48] Z. Qu, J. Wang, and R. A. Hull, "Cooperative control of dynamical systems with application to autonomous vehicles," IEEE Transactions on Automatic Control, vol. 53, no. 4, pp. 894-911, May 2008.

[49] S. E. Tuna, "Conditions for synchronizability in arrays of coupled linear systems," IEEE Transactions on Automatic Control, vol. 54, no. 10, pp. 2416-2420, October 2009.

[50] L. Scardovi and R. Sepulchre, "Synchronization in networks of identical linear systems," Automatica, vol. 45, no. 11, pp. 2557-2562, 2009.

[51] J. H. Seo, H. Shim, and J. Back, "Consensus of high-order linear systems using dynamic output feedback compensator: Low gain approach," Automatica, vol. 45, no. 11, pp. 2659-2664, 2009.

[52] Z. Li, Z. Duan, G. Chen, and L. Huang, "Consensus of multiagent systems and synchronization of complex networks: A unified viewpoint," IEEE Transactions on Circuits And SystemsI: Regular Papers, vol. 57, no. 1, pp. 213-224, 2010.

[53] T. Yang, S. Roy, Y. Wan, and A. Saberi, "Constructing consensus controllers for networks with identical general linear agents," International Journal of Robust and Nonlinear Control, vol. 21, no. 11, pp. 1237-1256, 2011.

[54] G.-B. Stan and R. Sepulchre, "Analysis of interconnected oscillators by dissipativity theory," IEEE Transactions on Automatic Control, vol. 52, no. 2, pp. 256-270, February 2007.

[55] Z. Lin, B. Francis, and M. Maggiore, "State agreement for continuous-time coupled nonlinear systems," SIAM Journal on Control and Optimization, vol. 46, no. 1, pp. 288-307, 2007.

[56] D. V. Dimarogonas and K. J. Kyriakopoulos, "A connection between formation infeasibility and velocity alignment in kinematic multi-agent systems," Automatica, vol. 44, no. 10, pp. 2648-2654, 2008.

[57] J. Zhou, J.-A. Lu, and J. Lü, “Adaptive synchronization of an uncertain complex dynamical network," IEEE Transactions on Automatic Control, vol. 51, no. 4, pp. 652-656, April 2006.

[58] — - "Pinning adaptive synchronization of a general complex dynamical network," Automatica, vol. 44, no. 4, pp. 9961003, 2008.

[59] N. Chopra and M. W. Spong, "On exponential synchronization of kuramoto oscillators," IEEE Transactions on Automatic Control, vol. 54, no. 2, pp. 353-357, February 2009. 
[60] W. Yu, J. Cao, G. Chen, J. Lü, J. Han, and W. Wei, "Local synchronization of a complex network model," IEEE Transactions on Systems, Man, and Cybernetics, Part B: Cybernetics, vol. 39, no. 1, pp. 230-241, February 2009.

[61] F. Chen, Z. Chen, L. Xiang, Z. Liu, and Z. Yuan, "Reaching a consensus via pinning control," Automatica, vol. 45, no. 5, pp. 1215-1220, 2009.

[62] Z.-G. Hou, L. Cheng, and M. Tan, "Decentralized robust adaptive control for the multiagent system consensus problem using neural networks," IEEE Transactions on Systems, Man, and Cybernetics, Part B: Cybernetics, vol. 39, no. 3, pp. 636-647, June 2009.

[63] P. DeLellis, M. diBernardo, and F. Garofalo, "Novel decentralized adaptive strategies for the synchronization of complex networks," Automatica, vol. 45, no. 5, pp. 1312-1318, 2009.

[64] G. Shi and Y. Hong, "Global target aggregation and state agreement of nonlinear multi-agent systems with switching topologies," Automatica, vol. 45, no. 5, pp. 1165-1175, 2009.

[65] A. Sarlette, R. Sepulchre, and N. E. Leonard, "Autonomous rigid body attitude synchronization," Automatica, vol. 45, no. 2, pp. 572-577, 2009.

[66] H. Bai, M. Arcak, and J. T. Wen, "Rigid body attitude coordination without inertial frame information," Automatica, vol. 44, no. 12, pp. 3170-3175, 2008.

[67] S.-J. Chung and J.-J. E. Slotine, "Cooperative robot control and concurrent synchronization of lagrangian systems," IEEE Transactions on Robotics, vol. 25, no. 3, pp. 686-700, June 2009.

[68] S. Nair and N. E. Leonard, "Stable synchronization of mechanical system networks," SIAM Journal on Control and Optimization, vol. 47, no. 2, pp. 661-683, 2008.

[69] J. Yao, Z.-H. Guan, and D. J. Hill, "Passivity-based control and synchronization of general complex dynamical networks," Automatica, vol. 45, no. 9, pp. 2107-2113, 2009.

[70] J. Zhao, D. J. Hill, and T. Liu, "Synchronization of complex dynamical networks with switching topology: A switched system point of view," Automatica, vol. 45, no. 11, pp. 2502-2511, 2009.

[71] P. P. Menon and C. Edwards, "Decentralised static output feedback stabilisation and synchronisation of networks," Automatica, vol. 45, no. 12, pp. 2910-2916, 2009.

[72] Y.-W. Wang, H. O. Wang, J.-W. Xiao, and Z.-H. Guan, "Synchronization of complex dynamical networks under recoverable attacks," Automatica, vol. 46, no. 1, pp. 197-203, 2010.

[73] J. Lu, D. W. Ho, and J. Cao, “A unified synchronization criterion for impulsive dynamical networks," Automatica, vol. 46, no. 7, pp. 1215-1221, 2010.

[74] E. Estrada, S. Gago, and G. Caporossi, "Design of highly synchronizable and robust networks," Automatica, vol. 46, no. 11, pp. 1835-1842, 2010.

[75] A. Das and F. L. Lewis, "Distributed adaptive control for synchronization of unknown nonlinear networked systems," Automatica, vol. 46, no. 12, pp. 2014-2021, 2010.

[76] H. Su, G. Chen, X. Wang, and Z. Lin, "Adaptive second-order consensus of networked mobile agents with nonlinear dynamics," Automatica, vol. 47, no. 2, pp. 368-375, 2011.

[77] W. Yu, G. Chen, and M. Cao, "Consensus in directed networks of agents with nonlinear dynamics," IEEE Transactions on Automatic Control, vol. 56, no. 6, pp. 1436-1441, June 2011.

[78] L. Scardovi, M. Arcak, and E. D. Sontag, "Synchronization of interconnected systems with applications to biochemical networks: An input-output approach," IEEE Transactions on Automatic Control, vol. 55, no. 6, pp. 1367-1379, June 2010. 
[79] Q. Li and Z.-P. Jiang, "Global analysis of multi-agent systems based on vicsek's model," IEEE Transactions on Automatic Control, vol. 54, no. 12, pp. 2876-2881, December 2009.

[80] A. Abdessameud and A. Tayebi, "Attitude synchronization of a group of spacecraft without velocity measurements," IEEE Transactions on Automatic Control, vol. 54, no. 11, pp. 2642-2648, November 2009.

[81] G. Chen and F. L. Lewis, "Distributed adaptive tracking control for synchronization of unknown networked lagrangian systems," IEEE Transactions on Systems, Man, and Cybernetics, Part B: Cybernetics, vol. 41, no. 3, pp. 805-816, June 2011.

[82] H. Zhang, T. Ma, G.-B. Huang, and Z. Wang, "Robust global exponential synchronization of uncertain chaotic delayed neural networks via dual-stage impulsive control," IEEE Transactions on Systems, Man, and Cybernetics, Part B: Cybernetics, vol. 40, no. 3, pp. 831-844, June 2010.

[83] J. Lu and D. W. C. Ho, "Globally exponential synchronization and synchronizability for general dynamical networks," IEEE Transactions on Systems, Man, and Cybernetics, Part B: Cybernetics, vol. 40, no. 2, pp. 350-361, April 2010.

[84] L. Wang and X. Wang, "New conditions for synchronization in dynamical communication networks," Systems and Control Letters, vol. 60, no. 4, pp. 219-225, 2011.

[85] N. D. Powel and K. A. Morgansen, "Communication-based performance bounds in nonlinear coordinated control," International Journal of Robust and Nonlinear Control, vol. 21, no. 12, pp. 1410-1420, 2011.

[86] Y.-W. Wang, J.-W. Xiao, and H. O. Wang, "Global synchronization of complex dynamical networks with network failures," International Journal of Robust and Nonlinear Control, vol. 20, no. 15, pp. 1667-1677, 2010.

[87] W. Ren, "Distributed leaderless consensus algorithms for networked EulerLagrange systems," International Journal of Control, vol. 82, no. 11, pp. 2137-2149, 2009.

[88] Q. Hui, W. M. Haddad, and S. P. Bhat, "Finite-time semistability and consensus for nonlinear dynamical networks," IEEE Transactions on Automatic Control, vol. 53, no. 8, pp. 1887-1890, September 2008.

[89] Q. Hui and W. M. Haddad, "Distributed nonlinear control algorithms for network consensus," Automatica, vol. 44, no. 9, pp. 2375-2381, 2008.

[90] J. Cortes, "Finite-time convergent gradient flows with applications to network consensus," Automatica, vol. 42, no. 11, pp. 1993-2000, 2006.

[91] W. Yu, G. Chen, and J. Lü, “On pinning synchronization of complex dynamical networks,” Automatica, vol. 45, no. 2, pp. 429-435, 2009.

[92] D. V. Dimarogonas and K. J. Kyriakopoulos, "On the rendezvous problem for multiple nonholonomic agents," IEEE Transactions on Automatic Control, vol. 52, no. 5, pp. 916-922, May 2007.

[93] J. Cortes, "Distributed algorithms for reaching consensus on general functions," Automatica, vol. 44, no. 3, pp. 726-737, 2008.

[94] H. Bai, M. Arcak, and J. T. Wen, "Adaptive motion coordination: Using relative velocity feedback to track a reference velocity," Automatica, vol. 45, no. 4, pp. 1020-1025, 2009.

[95] X. Wang and G. Chen, "Synchronization in scale-free dynamical networks: robustness and fragility," IEEE Transactions on Circuits And SystemsI: Regular Papers, vol. 49, no. 1, pp. 54-62, 2002.

[96] — - "Synchronization in small-world dynamical networks," International Journal of Bifurcation and Chaos, vol. 12, no. 1, pp. 187-192, 2002.

[97] W. Yu, J. Cao, and J. Lü, "Global synchronization of linearly hybrid coupled networks with time-varying delay," SIAM Journal on Applied Dynamical Systems, vol. 7, no. 1, pp. 108-133, 2008. 
[98] C. Godsil and G. Royle, Algebraic Graph Theory. New York: Springer-Verlag, 2001.

[99] W. Ren and E. Atkins, "Distributed multi-vehicle coordinated control via local information exchange," International Journal of Robust and Nonlinear Control, vol. 17, no. 11, pp. 1002-1033, July 2007.

[100] W. Yu, G. Chen, and M. Cao, "Some necessary and sufficient conditions for second-order consensus in multi-agent dynamical systems," Automatica, vol. 46, no. 6, pp. 1089-1095, 2010.

[101] W. Yu, G. Chen, M. Cao, , and J. Kurths, "Second-order consensus for multiagent systems with directed topologies and nonlinear dynamics," IEEE Transactions on Systems, Man, and Cybernetics, Part B: Cybernetics, vol. 40, no. 3, pp. 881-891, 2010.

[102] W. Yu, G. Chen, W. Ren, J. Kurths, and W. X. Zheng, "Distributed higher order consensus protocols in multiagent dynamical systems," IEEE Transactions on Circuits And SystemsI: Regular Papers, vol. 58, no. 8, pp. 1924-1932, 2011.

[103] C.-Q. Ma and J.-F. Zhang, "Necessary and sufficient conditions for consensusability of linear multi-agent systems," IEEE Transactions on Automatic Control, vol. 55, no. 5, pp. 1263-1268, May 2010.

[104] W. Wang and J.-J. E. Slotine, "Contraction analysis of time-delayed communications and group cooperation," IEEE Transactions on Automatic Control, vol. 51, no. 4, pp. 712-717, April 2006.

[105] F. Xiao and L. Wang, "Consensus protocols for discrete-time multi-agent systems with time-varying delays," Automatica, vol. 44, no. 10, pp. 2577-2582, 2008.

[106] —_ "Asynchronous consensus in continuous-time multi-agent systems with switching topology and time-varying delays," IEEE Transactions on Automatic Control, vol. 53, no. 8, pp. 1804-1816, September 2008.

[107] Y. G. Sun, L. Wang, and G. Xie, "Average consensus in networks of dynamic agents with switching topologies and multiple time-varying delays," Systems and Control Letters, vol. 57, no. 2, pp. 175-183, 2008.

[108] P. Lin, Y. Jia, and L. Li, "Distributed robust $H_{\infty}$ consensus control in directed networks of agents with time-delay," Systems and Control Letters, vol. 57, no. 8, pp. 643-653, 2008.

[109] Y.-P. Tian and C.-L. Liu, "Consensus of multi-agent systems with diverse input and communication delays," IEEE Transactions on Automatic Control, vol. 53, no. 9, pp. 2122-2128, October 2008.

[110] Y. G. Sun and L. Wang, "Consensus of multi-agent systems in directed networks with nonuniform time-varying delays," IEEE Transactions on Automatic Control, vol. 54, no. 7, pp. 1607-1613, July 2009.

[111] U. Munz, A. Papachristodoulou, and F. Allgower, "Delay robustness in consensus problems," Automatica, vol. 46, no. 8, pp. 1252-1265, 2010.

[112] X. Liu, W. Lu, and T. Chen, "Consensus of multi-agent systems with unbounded time-varying delays," IEEE Transactions on Automatic Control, vol. 55, no. 10, pp. 2396-2401, October 2010.

[113] A. Papachristodoulou, A. Jadbabaie, and U. Munz, "Effects of delay in multi-agent consensus and oscillator synchronization," IEEE Transactions on Automatic Control, vol. 55, no. 6, pp. 1471-1477, June 2010.

[114] P. Lin and Y. Jia, "Multi-agent consensus with diverse time-delays and jointly-connected topologies," Automatica, vol. 47, no. 4, pp. 848-856, 2011.

[115] Y.-P. Tian and C.-L. Liu, "Robust consensus of multi-agent systems with diverse input delays and asymmetric interconnection perturbations," Automatica, vol. 45, no. 5, pp. 1347-1353, 2009.

[116] P. Lin and Y. Jia, "Consensus of second-order discrete-time multi-agent systems with nonuniform time-delays and dynamically changing topologies," Automatica, vol. 45, no. 9, pp. 2154-2158, 2009.

[117] B. Yang and H. Fang, "Forced consensus in networks of double integrator systems with delayed input," Automatica, vol. 46, no. 3, pp. 629-632, 2010.

July 31, 2011

DRAFT 
[118] Y. Zhang and Y.-P. Tian, "Consensus of data-sampled multi-agent systems with random communication delay and packet loss," IEEE Transactions on Automatic Control, vol. 55, no. 4, pp. 939-943, April 2010.

[119] P. Lin and Y. Jia, "Consensus of a class of second-order multi-agent systems with time-delay and jointly-connected topologies," IEEE Transactions on Automatic Control, vol. 55, no. 3, pp. 778-784, March 2010.

[120] F. Xiao, L. Wang, and J. Chen, "Partial state consensus for networks of second-order dynamic agents," Systems and Control Letters, vol. 59, no. 12, pp. 775-781, 2010.

[121] U. Munz, A. Papachristodoulou, and F. Allgower, "Robust consensus controller design for nonlinear relative degree two multi-agent systems with communication constraints," IEEE Transactions on Automatic Control, vol. 56, no. 1, pp. 145-151, January 2011.

[122] J. Qin, H. Gao, and W. X. Zheng, "Second-order consensus for multi-agent systems with switching topology and communication delay," Systems and Control Letters, vol. 60, no. 6, pp. 390-397, 2011.

[123] J. Cao, G. Chen, and P. Li, "Global synchronization in an array of delayed neural networks with hybrid coupling," IEEE Transactions on Systems, Man, and Cybernetics, Part B: Cybernetics, vol. 38, no. 2, pp. 488-498, April 2008.

[124] J. Liang, Z. Wang, Y. Liu, and X. Liu, "Global synchronization control of general delayed discrete-time networks with stochastic coupling and disturbances," IEEE Transactions on Systems, Man, and Cybernetics, Part B: Cybernetics, vol. 38, no. 4, pp. 1073-1083, August 2008.

[125] J. Yao, H. O. Wang, Z.-H. Guan, and W. Xu, "Passive stability and synchronization of complex spatio-temporal switching networks with time delays," Automatica, vol. 45, no. 7, pp. 1721-1728, 2009.

[126] Y. Wang, H. Zhang, X. Wang, and D. Yang, "Networked synchronization control of coupled dynamic networks with timevarying delay," IEEE Transactions on Systems, Man, and Cybernetics, Part B: Cybernetics, vol. 40, no. 6, pp. 1468-1479, December 2010 .

[127] N. Chopra, M. W. Spong, and R. Lozano, "Synchronization of bilateral teleoperators with time delay," Automatica, vol. 44, no. 8, pp. 2142-2148, 2008.

[128] E. Nuno, R. Ortega, L. Basanez, and D. Hill, "Synchronization of networks of nonidentical euler-lagrange systems with uncertain parameters and communication delays," IEEE Transactions on Automatic Control, vol. 56, no. 4, pp. 935-941, April 2011.

[129] V. S. Bokharaie, O. Mason, and M. Verwoerd, "D-stability and delay-independent stability of homogeneous cooperative systems," IEEE Transactions on Automatic Control, vol. 55, no. 12, pp. 2882-2885, December 2010.

[130] T. Hayakawa, T. Matsuzawa, and S. Hara, "Formation control of multi-agent systems with sampled information," in Proceedings of the IEEE Conference on Decision and Control, San Diego, CA, December 2006, pp. 4333-4338.

[131] G. Xie, H. Liu, L. Wang, and Y. Jia, "Consensus in networked multi-agent systems via sampled control: Fixed topology case," in Proceedings of the American Control Conference, St. Louis, MO, July 2009, pp. 3902-3907.

[132] _ - "Consensus in networked multi-agent systems via sampled control: Switching topology case," in Proceedings of the American Control Conference, St. Louis, MO, July 2009, pp. 4525-4530.

[133] Y. Gao, L. Wang, G. Xie, and B. Wu, "Consensus of multi-agent systems based on sampled-data control," International Journal of Control, vol. 82, no. 12, pp. 2193-2205, 2009.

[134] Y. Gao and L. Wang, "Consensus of multiple double-integrator agents with intermittent measurement," International Journal of Robust and Nonlinear Control, vol. 20, no. 10, pp. 1140-1155, 2010.

[135] Y. Cao and W. Ren, "Multi-vehicle coordination for double-integrator dynamics under fixed undirected/directed interaction in a sampled-data setting," International Journal of Robust and Nonlinear Control, vol. 20, pp. 987-1000, 2010.

July 31,2011

DRAFT 
[136] — - "Sampled-data discrete-time consensus algorithms for double-integrator dynamics under dynamic directed interaction,” International Journal of Control, vol. 83, pp. 506-515, 2010.

[137] W. Yu, W. X. Zheng, G. Chen, W. Ren, and J. Cao, "Second-order consensus in multi-agent dynamical systems with sampled position data," Automatica, vol. 47, no. 7, pp. 1496-1503, 2011.

[138] Y. Gao and L. Wang, "Sampled-data based consensus of continuous-time multi-agent systems with time-varying topology," IEEE Transactions on Automatic Control, vol. 56, no. 5, pp. 1226-1231, May 2011.

[139] H. Liu, G. Xie, and L. Wang, "Necessary and sufficient conditions for solving consensus problems of double-integrator dynamics via sampled control," International Journal of Robust and Nonlinear Control, vol. 20, no. 15, pp. 1706-1722, 2010.

[140] M. Cao, A. S. Morse, and B. D. O. Anderson, "Agreeing asynchronously," IEEE Transactions on Automatic Control, vol. 53, no. 8, pp. 1826-1838, September 2008.

[141] L. Fang and P. J. Antsaklis, "Asynchronous consensus protocols using nonlinear paracontractions theory," IEEE Transactions on Automatic Control, vol. 53, no. 10, pp. 2351-2355, November 2008.

[142] Y. Gao and L. Wang, "Asynchronous consensus of continuous-time multi-agent systems with intermittent measurements," International Journal of Control, vol. 83, no. 3, pp. 552-562, 2010.

[143] A. Kashyap, T. Basar, and R. Srikant, “Quantized consensus,” Automatica, vol. 43, no. 7, pp. 1192-1203, 2007.

[144] R. Carli and F. Bullo, "Quantized coordination algorithms for rendezvous and deployment," SIAM Journal on Control and Optimization, vol. 48, no. 3, pp. 1251-1274, 2009.

[145] J. Lavaei and R. M. Murray, "On quantized consensus by means of gossip algorithm Part I: Convergence proof," in Proceedings of the American Control Conference, St. Louis, MO, June 2009, pp. 394-401.

[146] _ _ "On quantized consensus by means of gossip algorithm-Part II: Convergence time," in Proceedings of the American Control Conference, St. Louis, MO, June 2009, pp. 2958-2965.

[147] D. Yuan, S. Xu, H. Zhao, and Y. Chu, "Distributed average consensus via gossip algorithm with real-valued and quantized data for $0<q<1$," Systems and Control Letters, vol. 59, no. 9, pp. 536-542, 2010.

[148] A. Nedic, A. Olshevsky, A. Ozdaglar, and J. N. Tsitsiklis, "On distributed averaging algorithms and quantization effects," IEEE Transactions on Automatic Control, vol. 54, no. 11, pp. 2506-2517, November 2009.

[149] M. Franceschelli, A. Giua, and C. Seatzu, "A gossip-based algorithm for discrete consensus over heterogeneous networks," IEEE Transactions on Automatic Control, vol. 55, no. 5, pp. 1244-1249, May 2010.

[150] T. Li, M. Fu, L. Xie, and J.-F. Zhang, "Distributed consensus with limited communication data rate," IEEE Transactions on Automatic Control, vol. 56, no. 2, pp. 279-292, February 2011.

[151] M. Zhu and S. Martinez, "On the convergence time of asynchronous distributed quantized averaging algorithms," IEEE Transactions on Automatic Control, vol. 56, no. 2, pp. 386-390, February 2011.

[152] R. Carli, G. Como, P. Frasca, and F. Garin, "Distributed averaging on digital erasure networks," Automatica, vol. 47, no. 1, pp. 115-121, 2011.

[153] D. V. Dimarogonas and K. H. Johansson, "Stability analysis for multi-agent systems using the incidence matrix: Quantized communication and formation control," Automatica, vol. 46, no. 4, pp. 695-700, 2010.

[154] R. Carli, F. Fagnani, P. Frasca, and S. Zampieri, "Gossip consensus algorithms via quantized communication,” Automatica, vol. 46, no. 1, pp. 70-80, 2010.

[155] Y. Kim and M. Mesbahi, "On maximizing the second smallest eigenvalue of a state-dependent graph laplacian," IEEE Transactions on Automatic Control, vol. 51, no. 1, pp. 116-120, January 2006.

July 31,2011

DRAFT 
[156] L. Xiao and S. Boyd, "Fast linear iterations for distributed averaging," Systems and Control Letters, vol. 53, no. 1, pp. 65-78, 2004.

[157] A. Olshevsky and J. N. Tsitsiklis, “Convergence speed in distributed consensus and averaging," SIAM Journal on Control and Optimization, vol. 48, no. 1, pp. 33-55, 2009.

[158] D. Angeli and P.-A. Bliman, "Convergence speed of unsteady distributed consensus: Decay estimate along the settling spanning-trees," SIAM Journal on Control and Optimization, vol. 48, no. 1, pp. 1-32, 2009.

[159] — - "Tight estimates for convergence of some non-stationary consensus algorithms," Systems and Control Letters, vol. 57, no. 12, pp. 996-1004, 2008.

[160] J. Zhou and Q. Wang, "Convergence speed in distributed consensus over dynamically switching random networks," Automatica, vol. 45, no. 6, pp. 1455-1461, 2009.

[161] F. Xiao, L. Wang, J. Chen, and Y. Gao, "Finite-time formation control for multi-agent systems," Automatica, vol. 45, no. 11, pp. 2605-2611, 2009.

[162] F. Jiang and L. Wang, "Finite-time information consensus for multi-agent systems with fixed and switching topologies," Physica D, vol. 238, no. 16, pp. 1550-1560, 2009.

[163] Y. Cao, W. Ren, and Z. Meng, "Decentralized finite-time sliding mode estimators and their applications in decentralized finite-time formation tracking," Systems and Control Letters, vol. 59, no. 9, pp. 522-529, 2010.

[164] L. Wang and F. Xiao, "Finite-time consensus problems for networks of dynamic agents," IEEE Transactions on Automatic Control, vol. 55, no. 4, pp. 950-955, April 2010.

[165] X. Wang and Y. Hong, "Finite-time consensus for multi-agent networks with second-order agent dynamics," in IFAC World Congress, Soeul, Korea, July 2008, pp. 15 185-15190.

[166] M. Pavone and E. Frazzoli, "Decentralized policies for geometric pattern formation and path coverage," ASME Journal of Dynamic Systems, Measurement, and Control, vol. 129, pp. 633-643, 2007.

[167] W. Ren, "Collective motion from consensus with cartesian coordinate coupling," IEEE Transactions on Automatic Control, vol. 54, no. 6, pp. 1330-1336, June 2009.

[168] P. Lin and Y. Jia, "Distributed rotating formation control of multi-agent systems," Systems and Control Letters, vol. 59, no. 10 , pp. $587-595,2010$.

[169] P. Lin, K. Qin, Z. Li, and W. Ren, "Collective rotating motions of second-order multi-agent systems in three-dimensional space," Systems and Control Letters, vol. 60, no. 6, pp. 365-372, 2011.

[170] R. Sepulchre, D. A. Paley, and N. E. Leonard, "Stabilization of planar collective motion: All-to-all communication," IEEE Transactions on Automatic Control, vol. 52, no. 5, pp. 811-824, May 2007.

[171] — - "Stabilization of planar collective motion with limited communication," IEEE Transactions on Automatic Control, vol. 53, no. 3, pp. 706-719, April 2008.

[172] D. V. Dimarogonas and K. J. Kyriakopoulos, "Inverse agreement protocols with application to distributed multi-agent dispersion," IEEE Transactions on Automatic Control, vol. 54, no. 3, pp. 657-663, March 2009.

[173] R. Olfati-Saber, "Flocking for multi-agent dynamic systems: Algorithms and theory," IEEE Transactions on Automatic Control, vol. 51, no. 3, pp. 401-420, March 2006.

[174] K. D. Do, "Bounded controllers for formation stabilization of mobile agents with limited sensing ranges," IEEE Transactions on Automatic Control, vol. 52, no. 3, pp. 569-576, March 2007.

[175] F. Cucker and S. Smale, "Emergent behavior in flocks," IEEE Transactions on Automatic Control, vol. 52, no. 5, pp. 852-862, May 2007.

July 31,2011

DRAFT 
[176] H. G. Tanner, A. Jadbabaie, and G. J. Pappas, "Flocking in fixed and switching networks," IEEE Transactions on Automatic Control, vol. 52, no. 5, pp. 863-868, May 2007.

[177] L. E. Barnes, M. A. Fields, and K. P. Valavanis, "Swarm formation control utilizing elliptical surfaces and limiting functions," IEEE Transactions on Systems, Man, and Cybernetics, Part B: Cybernetics, vol. 39, no. 6, pp. 1434-1445, December 2009.

[178] M. Kumar, D. P. Garg, and V. Kumar, "Segregation of heterogeneous units in a swarm of robotic agents," IEEE Transactions on Automatic Control, vol. 55, no. 3, pp. 743-748, March 2010.

[179] J. Park, H. J. Kim, and S.-Y. Ha, "Cucker-smale flocking with inter-particle bonding forces," IEEE Transactions on Automatic Control, vol. 55, no. 11, pp. 2617-2623, November 2010.

[180] S.-Y. Ha, T. Ha, and J.-H. Kim, "Emergent behavior of a cucker-smale type particle model with nonlinear velocity couplings," IEEE Transactions on Automatic Control, vol. 55, no. 7, pp. 1679-1683, July 2010.

[181] F. Cucker and J.-G. Dong, "Avoiding collisions in flocks," IEEE Transactions on Automatic Control, vol. 55, no. 5, pp. 1238-1243, May 2010.

[182] —-, "A general collision-avoiding flocking framework," IEEE Transactions on Automatic Control, vol. 56, no. 5, pp. 1124-1129, May 2011.

[183] H.-T. Zhang, C. Zhai, and Z. Chen, "A general alignment repulsion algorithm for flocking of multi-agent systems," IEEE Transactions on Automatic Control, vol. 56, no. 2, pp. 430-435, February 2011.

[184] F. Zhang and N. E. Leonard, "Coordinated patterns of unit speed particles on a closed curve," Systems and Control Letters, vol. 56, no. 6, pp. 397-407, 2007.

[185] D. A. Paley, "Stabilization of collective motion on a sphere," Automatica, vol. 45, no. 1, pp. 212-216, 2009.

[186] R. Mellish, S. Napora, and D. A. Paley, "Backstepping control design for motion coordination of self-propelled vehicles in a flowfield," International Journal of Robust and Nonlinear Control, vol. 21, no. 12, pp. 1452-1466, 2011.

[187] S. Hernandez and D. A. Paley, "Three-dimensional motion coordination in a spatiotemporal flowfield," IEEE Transactions on Automatic Control, vol. 55, no. 12, pp. 2805-2810, December 2010.

[188] D. A. Paley, N. E. Leonard, and R. Sepulchre, "Stabilization of symmetric formations to motion around convex loops," Systems and Control Letters, vol. 57, no. 3, pp. 209-215, 2008.

[189] G. Laman, "On graphs and rigidity of plane skeletal structures," Journal of Engineering Mathematics, vol. 4, no. 4, pp. 331-340, 1970.

[190] R. Olfati-Saber and R. M. Murray, "Graph rigidity and distributed formation stabilization of multi-vehicle systems," in Proceedings of the IEEE Conference on Decision and Control, Las Vegas, NV, December 2002, pp. $2965-2971$.

[191] J. M. Hendrickx, B. D. O. Anderson, J.-C. Delvenne, and V. D. Blondel, "Directed graphs for the analysis of rigidity and persistence in autonomous agent systems," International Journal of Robust and Nonlinear Control, vol. 17, no. 11, pp. 960-981, July 2007.

[192] C. Yu, B. D. O. Anderson, S. Dasgupta, and B. Fidan, "Control of minimally persistent formations in the plane," SIAM Journal on Control and Optimization, vol. 48, no. 1, pp. 206-233, 2009.

[193] B. Fidan, J. M. Hendrickx, and B. D. O. Anderson, "Closing ranks in rigid multi-agent formations using edge contraction," International Journal of Robust and Nonlinear Control, vol. 20, no. 18, pp. 2077-2092, 2010.

[194] M. Cao, C. Yu, and B. D. Anderson, "Formation control using range-only measurements," Automatica, vol. 47, no. 4, pp. 776-781, 2011. 
[195] W. B. Dunbar and R. M. Murray, "Distributed receding horizon control for multi-vehicle formation stabilization," Automatica, vol. 42, no. 4, pp. 549-558, 2006.

[196] W. B. Dunbar, "Distributed receding horizon control of dynamically coupled nonlinear systems," IEEE Transactions on Automatic Control, vol. 52, no. 7, pp. 1249-1263, July 2007.

[197] E. Franco, L. Magni, T. Parisini, M. M. Polycarpou, and D. M. Raimondo, "Cooperative constrained control of distributed agents with nonlinear dynamics and delayed information exchange: A stabilizing receding-horizon approach," IEEE Transactions on Automatic Control, vol. 53, no. 1, pp. 324-338, February 2008.

[198] W. Ren, "Multi-vehicle consensus with a time-varying reference state," Systems and Control Letters, vol. 56, no. 7, pp. 474-483, 2007.

[199] Y. Cao, W. Ren, and Y. Li, "Distributed discrete-time coordinated tracking with a time-varying reference state and limited communication," Automatica, vol. 45, no. 5, pp. 1299-1305, 2009.

[200] J. Xiang, W. Wei, and Y. Li, "Synchronized output regulation of linear networked systems," IEEE Transactions on Automatic Control, vol. 54, no. 6, pp. 1336-1341, June 2009.

[201] X. Wang, Y. Hong, J. Huang, and Z.-P. Jiang, "A distributed control approach to a robust output regulation problem for multi-agent linear systems," IEEE Transactions on Automatic Control, vol. 55, no. 12, pp. 2891-2895, December 2010.

[202] M. Porfiri, D. G. Roberson, and D. J. Stilwell, "Tracking and formation control of multiple autonomous agents:a two-level consensus approach," Automatica, vol. 43, no. 8, pp. 1318-1328, 2007.

[203] H. Su, X. Wang, and Z. Lin, "Flocking of multi-agents with a virtual leader," IEEE Transactions on Automatic Control, vol. 54, no. 2, pp. 293-307, February 2009.

[204] H. Shi, L. Wang, and T. Chu, "Flocking of multi-agent systems with a dynamic virtual leader," International Journal of Control, vol. 82, no. 1, pp. 43-58, January 2009.

[205] W. Yu, G. Chen, and M. Cao, "Distributed leader-follower flocking control for multi-agent dynamical systems with time-varying velocities," Systems and Control Letters, vol. 59, no. 9, pp. 543-552, 2010.

[206] Y. Cao and W. Ren, "Distributed coordinated tracking with reduced interaction via a variable structure approach," IEEE Transactions on Automatic Control, 2011, to appear.

[207] W. Ni and D. Cheng, "Leader-following consensus of multi-agent systems under fixed and switching topologies," Systems and Control Letters, vol. 59, no. 3-4, pp. 209-217, 2010.

[208] W. Ren, "Consensus tracking under directed interaction topologies: Algorithms and experiments," IEEE Transactions on Control Systems Technology, vol. 18, no. 1, pp. 230-237, January 2010.

[209] J. Hu and G. Feng, "Distributed tracking control of leader-follower multi-agent systems under noisy measurement," Automatica, vol. 46, no. 8, pp. 1382-1387, 2010.

[210] W. Wang and J.-J. E. Slotine, “A theoretical study of different leader roles in networks," IEEE Transactions on Automatic Control, vol. 51, no. 7, pp. 1156-1161, July 2006.

[211] Q. Song, J. Cao, and W. Yu, "Second-order leader-following consensus of nonlinear multi-agent systems via pinning control," Systems and Control Letters, vol. 59, no. 9, pp. 553-562, 2010.

[212] K. D. Do, "Formation tracking control of unicycle-type mobile robots with limited sensing ranges," IEEE Transactions on Control Systems Technology, vol. 16, no. 3, pp. 527-538, May 2008.

[213] T. Gustavi and X. Hu, "Observer-based leader-following formation control using onboard sensor information," IEEE Transactions on Robotics, vol. 24, no. 6, pp. 1457-1462, December 2008.

July 31, 2011

DRAFT 
[214] W. Dong and J. A. Farrell, “Cooperative control of multiple nonholonomic mobile agents," IEEE Transactions on Automatic Control, vol. 53, no. 6, pp. 1434-1448, July 2008.

[215] — - "Decentralized cooperative control of multiple nonholonomic dynamic systems with uncertainty," Automatica, vol. 45, no. 3, pp. 706-710, 2009.

[216] W. Dong, "Flocking of multiple mobile robots based on backstepping," IEEE Transactions on Systems, Man, and Cybernetics, Part B: Cybernetics, vol. 41, no. 2, pp. 414-514, April 2011.

[217] E. J. Rodriguez-Seda, J. J. Troy, C. A. Erignac, P. Murray, D. M. Stipanovic, and M. W. Spong, "Bilateral teleoperation of multiple mobile agents: Coordinated motion and collision avoidance," IEEE Transactions on Control Systems Technology, vol. 18, no. 4, pp. 984-992, July 2010.

[218] W. Ren, "Distributed cooperative attitude synchronization and tracking for multiple rigid bodies," IEEE Transactions on Control Systems Technology, vol. 18, no. 2, pp. 383-392, March 2010.

[219] H. Su, X. Wang, and G. Chen, "Rendezvous of multiple mobile agents with preserved network connectivity," Systems and Control Letters, vol. 59, no. 5, pp. 313-322, 2010.

[220] M. M. Zavlanos, H. G. Tanner, A. Jadbabaie, and G. J. Pappas, "Hybrid control for connectivity preserving flocking," IEEE Transactions on Automatic Control, vol. 54, no. 12, pp. 2869-2875, December 2009.

[221] A. Ajorlou, A. Momeni, and A. G. Aghdam, "A class of bounded distributed control strategies for connectivity preservation in multi-agent systems," IEEE Transactions on Automatic Control, vol. 55, no. 12, pp. 2828-2833, December 2010.

[222] T. Gustavi, D. V. Dimarogonas, M. Egerstedt, and X. Hu, "Sufficient conditions for connectivity maintenance and rendezvous in leader-follower networks," Automatica, vol. 46, no. 1, pp. 133-139, 2010.

[223] H. Su, X. Wang, and Z. Lin, "Synchronization of coupled harmonic oscillators in a dynamic proximity network," Automatica, vol. 45, no. 10, pp. 2286-2291, 2009.

[224] H. Su, X. Wang, and G. Chen, "A connectivity-preserving flocking algorithm for multi-agent systems based only on position measurements," International Journal of Control, vol. 82, no. 7, pp. 1334-1343, July 2009.

[225] M. Ji and M. Egerstedt, "Distributed coordination control of multiagent systems while preserving connectedness," IEEE Transactions on Robotics, vol. 23, no. 4, pp. 693-703, August 2007.

[226] M. M. Zavlanos and G. J. Pappas, "Distributed connectivity control of mobile networks," IEEE Transactions on Robotics, vol. 24, no. 6, pp. 1416-1428, December 2008.

[227] B. Shucker, T. D. Murphey, and J. K. Bennett, "Convergence-preserving switching for topology-dependent decentralized systems," IEEE Transactions on Robotics, vol. 24, no. 6, pp. 1405-1415, December 2008.

[228] M. M. Zavlanos and G. J. Pappas, "Potential fields for maintaining connectivity of mobile networks," IEEE Transactions on Robotics, vol. 23, no. 4, pp. 812-816, August 2007.

[229] Z. Sun and J. Huang, "A note on connectivity of multi-agent systems with proximity graphs and linear feedback protocol," Automatica, vol. 45, no. 8, pp. 1953-1956, 2009.

[230] Y. Kim, "Bisection algorithm of increasing algebraic connectivity by adding an edge," IEEE Transactions on Automatic Control, vol. 55, no. 1, pp. 170-174, January 2010.

[231] R. Carli, A. Chiuso, L. Schenato, and S. Zampieri, "Optimal synchronization for networks of noisy double integrators," IEEE Transactions on Automatic Control, vol. 56, no. 5, pp. 1146-1152, May 2011.

[232] Y. Kim, D.-W. Gu, and I. Postlethwaite, "Spectral radius minimization for optimal average consensus and output feedback stabilization," Automatica, vol. 45, no. 6, pp. 1379-1386, 2009.

July 31,2011

DRAFT 
[233] M. Rabbat and R. Nowak, "Distributed optimization in sensor networks," in ACM Information Processing in Sensor Networks, Berkeley, CA, April 2004, pp. 20-27.

[234] A. Nedic and A. Ozdaglar, "Distributed subgradient methods for multi-agent optimization," IEEE Transactions on Automatic Control, vol. 54, no. 1, pp. 48-61, January 2009.

[235] A. Nedic, A. Ozdaglar, and P. A. Parrilo, "Constrained consensus and optimization in multi-agent networks," IEEE Transactions on Automatic Control, vol. 55, no. 4, pp. 922-938, April 2010.

[236] I. Lobel and A. Ozdaglar, "Distributed subgradient methods for convex optimization over random networks," IEEE Transactions on Automatic Control, vol. 56, no. 6, pp. 1291-1306, June 2011.

[237] A. Nedic, "Asynchronous broadcast-based convex optimization over a network," IEEE Transactions on Automatic Control, vol. 56, no. 6, pp. 1337-1351, June 2011.

[238] Y. Cao and W. Ren, “Optimal linear consensus algorithms: An LQR perspective,” IEEE Transactions on Systems, Man, and Cybernetics, Part B: Cybernetics, vol. 40, pp. 819-830, 2010, 2010.

[239] F. Borrelli and T. Keviczky, "Distributed LQR design for identical dynamically decoupled systems," IEEE Transactions on Automatic Control, vol. 53, no. 8, pp. 1901-1912, September 2008.

[240] E. Semsar-Kazerooni and K. Khorasani, "Optimal consensus seeking in a network of multiagent systems: An LMI approach," IEEE Transactions on Systems, Man, and Cybernetics, Part B: Cybernetics, vol. 40, no. 2, pp. 540-547, April 2010.

[241] J. Wang and M. Xin, "Multi-agent consensus algorithm with obstacle avoidance via optimal control approach," International Journal of Control, vol. 83, no. 12, pp. 2606-2621, 2010.

[242] W. Dong, "Distributed optimal control of multiple systems," International Journal of Control, vol. 83, no. 10, pp. 20672079, 2010.

[243] J. Hu, M. Prandini, and C. Tomlin, "Conjugate points in formation constrained optimal multi-agent coordination: A case study," SIAM Journal on Control and Optimization, vol. 45, no. 6, pp. 2119-2137, 2006.

[244] E. Franco, T. Parisini, and M. M. Polycarpou, "Design and stability analysis of cooperative receding-horizon control of linear discrete-time agents," International Journal of Robust and Nonlinear Control, vol. 17, no. 11, pp. 982-1001, July 2007.

[245] G. Ferrari-Trecate, L. Galbusera, M. P. E. Marciandi, and R. Scattolini, "Model predictive control schemes for consensus in multi-agent systems with single- and double-integrator dynamics," IEEE Transactions on Automatic Control, vol. 54, no. 11, pp. 2560-2572, November 2009.

[246] B. T. Stewart, A. N. Venkat, J. B. Rawlings, S. J. Wright, and G. Pannocchia, "Cooperative distributed model predictive control," Systems and Control Letters, vol. 59, no. 8, pp. 460-469, 2010.

[247] J. Cortés, S. Martínez, T. Karatas, and F. Bullo, “Coverage control for mobile sensing networks," IEEE Transactions on Robotics and Automation, vol. 20, pp. 243-255, 2004.

[248] J. Cortes, S. Martinez, and F. Bullo, "Spatially distributed coverage optimization and control with limited-range interactions," ESAIM: Control, Optimisation and Calculus of Variations, vol. 11, no. 4, pp. 691-719, 2005.

[249] C. Gao, J. Cortes, and F. Bullo, "Notes on averaging over acyclic digraphs and discrete coverage control," Automatica, vol. 44, no. 8, pp. 2120-2127, 2008.

[250] K. Laventalla and J. Cortes, "Coverage control by multi-robot networks with limited-range anisotropic sensory," International Journal of Control, vol. 82, no. 6, pp. 1113-1121, June 2009.

July 31,2011

DRAFT 
[251] A. Kwok and S. Martinez, "A distributed deterministic annealing algorithm for limited-range sensor coverage," IEEE Transactions on Control Systems Technology, vol. 19, no. 4, pp. 792-804, July 2011.

[252] Y. Wang and I. I. Hussein, "Awareness coverage control over large-scale domains with intermittent communications," IEEE Transactions on Automatic Control, vol. 55, no. 8, pp. 1850-1859, August 2010.

[253] J. Choi and R. Horowitz, "Learning coverage control of mobile sensing agents in one-dimensional stochastic environments," IEEE Transactions on Automatic Control, vol. 55, no. 3, pp. 804-809, March 2010.

[254] J. Cortes, "Coverage optimization and spatial load balancing by robotic sensor networks," IEEE Transactions on Automatic Control, vol. 55, no. 3, pp. 749-754, March 2010.

[255] A. Kwok and S. Martinez, "Unicycle coverage control via hybrid modeling," IEEE Transactions on Automatic Control, vol. 55, no. 2, pp. 528-532, February 2010.

[256] Z. Jin, T. Shima, and C. J. Schumacher, "Optimal scheduling for refueling multiple autonomous aerial vehicles," IEEE Transactions on Robotics, vol. 22, no. 4, pp. 682-693, August 2006.

[257] J. Finke, K. M. Passino, and A. G. Sparks, "Stable task load balancing strategies for cooperative control of networked autonomous air vehicles," IEEE Transactions on Control Systems Technology, vol. 14, no. 5, pp. 789-803, September 2006.

[258] K. L. Moore and D. Lucarelli, "Decentralized adaptive scheduling using consensus variables," International Journal of Robust and Nonlinear Control, vol. 17, no. 11, pp. 921-940, July 2007.

[259] H.-L. Choi, L. Brunet, and J. P. How, "Consensus-based decentralized auctions for robust task allocation," IEEE Transactions on Robotics, vol. 25, no. 4, pp. 912-926, August 2009.

[260] S. A. Reveliotis and E. Roszkowska, "Conflict resolution in free-ranging multivehicle systems: A resource allocation paradigm," IEEE Transactions on Robotics, vol. 27, no. 2, pp. 283-296, April 2011.

[261] H. Sayyaadi and M. Moarref, "A distributed algorithm for proportional task allocation in networks of mobile agents," IEEE Transactions on Automatic Control, vol. 56, no. 2, pp. 405-410, February 2011.

[262] D. A. Anisi, P. Ogren, and X. Hu, "Cooperative minimum time surveillance with multiple ground vehicles," IEEE Transactions on Automatic Control, vol. 55, no. 12, pp. 2679-2691, December 2010.

[263] D. Kingston, R. W. Beard, and R. S. Holt, "Decentralized perimeter surveillance using a team of UAVs," IEEE Transactions on Robotics, vol. 24, no. 6, pp. 1394-1404, December 2008.

[264] B. J. Moore and K. M. Passino, "Decentralized redistribution for cooperative patrol," International Journal of Robust and Nonlinear Control, vol. 18, no. 2, pp. 165-195, January 2008.

[265] A. E. Gil, K. M. Passino, and J. B. Cruz, "Stable cooperative surveillance with information flow constraints," IEEE Transactions on Control Systems Technology, vol. 16, no. 5, pp. 856-868, September 2008.

[266] M. Peasgood, C. M. Clark, and J. McPhee, "A complete and scalable strategy for coordinating multiple robots within roadmaps," IEEE Transactions on Robotics, vol. 24, no. 2, pp. 283-292, April 2008.

[267] Y. Yang, M. M. Polycarpou, and A. A. Minai, "Multi-UAV cooperative search using an opportunistic learning method," ASME Journal of Dynamic Systems, Measurement, and Control, vol. 129, pp. 716-728, 2007.

[268] J. Finke and K. M. Passino, “Stable cooperative vehicle distributions for surveillance,” ASME Journal of Dynamic Systems, Measurement, and Control, vol. 129, pp. 597-608, 2007.

[269] Z. Tang and U. Ozguner, "Cooperative sensor deployment for multi-target monitoring," International Journal of Robust and Nonlinear Control, vol. 18, no. 2, pp. 196-217, January 2008.

July 31, 2011

DRAFT 
[270] F. Zhang and N. E. Leonard, “Cooperative filters and control for cooperative exploration," IEEE Transactions on Automatic Control, vol. 55, no. 3, pp. 650-663, March 2010.

[271] P. Yang, R. Freeman, G. Gordon, K. Lynch, S. Srinivasa, and R. Sukthankar, "Decentralized estimation and control of graph connectivity for mobile sensor networks," Automatica, vol. 46, no. 2, pp. 390-396, 2010.

[272] J. Choi, S. Oh, and R. Horowitz, "Distributed learning and cooperative control for multi-agent systems," Automatica, vol. 45, no. 12, pp. 2802-2814, 2009.

[273] K. M. Lynch, I. B. Schwartz, P. Yang, and R. A. Freeman, "Decentralized environmental modeling by mobile sensor networks," IEEE Transactions on Robotics, vol. 24, no. 3, pp. 710-724, June 2008.

[274] P. Yang, R. A. Freeman, and K. M. Lynch, "Multi-agent coordination by decentralized estimation and control," IEEE Transactions on Automatic Control, vol. 53, no. 11, pp. 2480-2496, December 2008.

[275] R. S. Smith and F. Y. Hadaegh, "Closed-loop dynamics of cooperative vehicle formations with parallel estimators and communication," IEEE Transactions on Automatic Control, vol. 52, no. 8, pp. 1404-1414, August 2007.

[276] S. S. Stankovic, M. S. Stankovic, and D. M. Stipanovic, "Consensus based overlapping decentralized estimation with missing observations and communication faults," Automatica, vol. 45, no. 6, pp. 1397-1406, 2009.

[277] G. C. Calafiore and F. Abrate, "Distributed linear estimation over sensor networks," International Journal of Control, vol. 82, no. 5, pp. 868-882, May 2009.

[278] M. A. Demetriou and I. I. Hussein, "Estimation of spatially distributed processes using mobile spatially distributed sensor network," SIAM Journal on Control and Optimization, vol. 48, no. 1, pp. 266-291, 2009.

[279] N. Marechal, J.-M. Gorce, and J.-B. Pierrot, "Joint estimation and gossip averaging for sensor network applications," IEEE Transactions on Automatic Control, vol. 55, no. 5, pp. 1208-1213, May 2010.

[280] S. S. Stankovic, M. S. Stankovic, and D. M. Stipanovic, "Decentralized parameter estimation by consensus based stochastic approximation," IEEE Transactions on Automatic Control, vol. 56, no. 3, pp. 531-543, March 2011.

[281] D. Yu, "Estimating the topology of complex dynamical networks by steady state control: Generality and limitation," Automatica, vol. 46, no. 12, pp. 2035-2040, 2010.

[282] M. V. Subbotin and R. S. Smith, "Design of distributed decentralized estimators for formations with fixed and stochastic communication topologies," Automatica, vol. 45, no. 11, pp. 2491-2501, 2009.

[283] H. Hirai and F. Miyazaki, "Dynamic coordination between robots: Self-organized timing selection in a juggling-like ballpassing task," IEEE Transactions on Systems, Man, and Cybernetics, Part B: Cybernetics, vol. 36, no. 4, pp. 738-754, August 2006.

[284] F. Kunwar and B. Benhabib, "Rendezvous-guidance trajectory planning for robotic dynamic obstacle avoidance and interception," IEEE Transactions on Systems, Man, and Cybernetics, Part B: Cybernetics, vol. 36, no. 6, pp. 1432-1441, December 2006.

[285] G. Antonelli and S. Chiaverini, "Kinematic control of platoons of autonomous vehicles," IEEE Transactions on Robotics, vol. 22, no. 6, pp. 1285-1292, December 2006.

[286] G. Baldassarre, V. Trianni, M. Bonani, F. Mondada, M. Dorigo, and S. Nolfi, "Self-organized coordinated motion in groups of physically connected robots," IEEE Transactions on Systems, Man, and Cybernetics, Part B: Cybernetics, vol. 37, no. 1, pp. 224-239, February 2007.

[287] W. Ren, H. Chao, W. Bourgeous, N. Sorensen, and Y. Chen, "Experimental validation of consensus algorithms for multivehicle cooperative control," IEEE Transactions on Control Systems Technology, vol. 16, no. 4, pp. 745-752, July 2008. 
[288] R. OGrady, A. L. Christensen, and M. Dorigo, "Swarmorph: Multirobot morphogenesis using directional self-assembly," IEEE Transactions on Robotics, vol. 25, no. 3, pp. 738-743, June 2009.

[289] Z. Cao, M. Tan, L. Li, N. Gu, and S. Wang, "Cooperative hunting by distributed mobile robots based on local interaction," IEEE Transactions on Robotics, vol. 22, no. 2, pp. 403-407, April 2006.

[290] S. D. Bopardikar, F. Bullo, and J. P. Hespanha, "A cooperative homicidal chauffeur game," Automatica, vol. 45, no. 7, pp. 1771-1777, 2009.

[291] — - "On discrete-time pursuit-evasion games with sensing limitations," IEEE Transactions on Robotics, vol. 24, no. 6, pp. 1429-1439, December 2008.

[292] A. Kolling and S. Carpin, "Pursuit-evasion on trees by robot teams," IEEE Transactions on Robotics, vol. 26, no. 1, pp. 32-47, February 2010.

[293] X. Liang and Y. Xiao, "Studying bio-inspired coalition formation of robots for detecting intrusions using game theory," IEEE Transactions on Systems, Man, and Cybernetics, Part B: Cybernetics, vol. 40, no. 3, pp. 683-693, June 2010.

[294] D. Gu, "A differential game approach to formation control," IEEE Transactions on Control Systems Technology, vol. 16, no. 1, pp. 85-93, January 2008.

[295] D. Bauso, L. Giarre, and R. Pesenti, "Consensus for networks with unknown but bounded disturbances," SIAM Journal on Control and Optimization, vol. 48, no. 3, pp. 1756-1770, 2009.

[296] —- "Consensus in noncooperative dynamic games: A multiretailer inventory application," IEEE Transactions on Automatic Control, vol. 53, no. 4, pp. 998-1003, May 2008.

[297] P. Vrancx, K. Verbeeck, and A. Nowe, "Decentralized learning in markov games," IEEE Transactions on Systems, Man, and Cybernetics, Part B: Cybernetics, vol. 38, no. 4, pp. 976-981, August 2008.

[298] M. Huang, P. E. Caines, and R. P. Malhame, "Large-population cost-coupled LQG problems with nonuniform agents: Individual-mass behavior and decentralized $\epsilon$-nash equilibria," IEEE Transactions on Automatic Control, vol. 52, no. 9, pp. 1560-1571, September 2007. 